\title{
Tuneable properties of carbon quantum dots by different synthetic methods
}

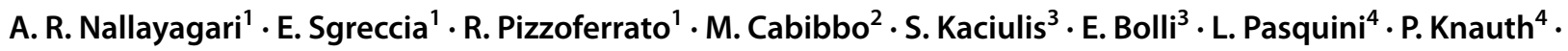 \\ M. L. Di Vona ${ }^{1}$ (i)
}

Received: 2 February 2021 / Accepted: 20 July 2021 / Published online: 30 July 2021

(c) The Author(s) 2021

\begin{abstract}
Carbon quantum dots (CQD) were prepared from three different precursors and by three bottom-up synthesis methods: classical pyrolysis of citric acid (CAP), microwave irradiation of glucose (GM), and hydrothermal treatment of glucosamine hydrochloride $(\mathrm{GAH})$. CQD were further functionalized using various nitrogen-containing compounds: 6-aminohexanoic acid, 1,6-diaminohexane, N-octylamine, dimethylamine, and tryptophan. Special attention was dedicated to investigate how the combination of synthetic method and starting material affected the nature and properties of CQD. The analysis indicated that CAP were good candidates for covalent post-functionalization, GM allowed an easy passivation, and GAH permitted the direct introduction of nitrogen into the core. The size distribution showed a core-shell structure for CQD functionalized with an aminoacid by microwave irradiation, whereas the thermal decomposition evidenced the degradation of functionalizing molecules and the presence of pyridinic and pyrrolic nitrogen after hydrothermal synthesis. Photoluminescence spectra revealed important differences between the synthesis techniques, related to the occurrence of surface states, and the highest fluorescence quantum yield for hydrothermally prepared CQD. These approaches led to CQD with properties that can be exploited in many fields from energy conversion to sensing.
\end{abstract}

Keywords Pyrolysis $\cdot$ Microwave irradiation $\cdot$ Hydrothermal treatment $\cdot$ Photoluminescence $\cdot$ Carbon nanodots

\section{Introduction}

Carbon quantum dots (CQD) are fascinating nanomaterials with tuneable band gap and high photoluminescence (PL) efficiency [1, 2]. The easy synthesis [3], the absence

P. Knauth

philippe.knauth@univ-amu.fr

$\triangle$ M. L. Di Vona

divona@uniroma2.it

1 Department of Industrial Engineering and International Laboratory: Ionomer Materials for Energy, University of Rome Tor Vergata, 00133 Roma, Italy

2 Department of Industrial Engineering and Mathematical Sciences (DIISM), Università Politecnica Delle Marche, Ancona, Italy

3 Institute for the Study of Nanostructured Materials, ISMN-CNR, 00015 Monterotondo Stazione, Roma, Italy

4 Aix Marseille Univ, CNRS, MADIREL (UMR 7246) and International Laboratory: Ionomer Materials for Energy, Campus St Jérôme, 13013 Marseille, France of toxicity and the huge variability by a vast number of functionalizing molecules [4] are very attractive features that explain the current excitement about CQD.

CQD are semiconducting or insulating particles depending on the nature of the functionalizing groups [5]. For example, nitrogen doping by urea [6] leads to a band gap decrease, because some donor nitrogen atoms are introduced into the graphene planes [7]. Given their semiconducting properties and environmental friendliness, CQD were proposed as noble-metal free catalytic electrodes for the oxygen reduction reaction (ORR) in fuel cells [8]. The emission ranges of photoluminescence can also be tuned by proper functionalization $[2,6,9,10]$ : for example, the blue photoluminescence of unfunctionalized CQD is shifted to green by amines $[9,11]$.

Various synthetic bottom-up methods were reported in the literature [12-15]. A simple pyrolysis approach was developed by carbonization of common organic molecules [16, 17]. Starting from citric acid (CA), CQD and graphene oxide (GO) can be obtained tuning the carbonization degree of the precursor. Depending on the carbonization temperature or 
time, CA can be incompletely carbonized affording CQD with a strong photoluminescence activity or, after complete pyrolysis, GO with a weak and excitation-dependent PL activity $[17,18]$. The application of this method is to some extent challenging, since the structure of CQD cannot be exactly controlled due to carbonaceous aggregation during carbonization [19]. Furthermore, pyrolysis is a time-taking process and a careful monitoring of the reaction is needed.

Microwave irradiation is another effective method to prepare CQD by providing a homogeneous heat distribution to the precursor solution, which quickens the formation of CQD and saves time. Zhu et al. synthesized CQD by treating poly(ethylene glycol) and saccharide solutions for few minutes in a microwave oven [14]. Chandra et al. prepared green fluorescent CQD from sucrose and phosphoric acid in a short time [20]. Advantages of this method include the ability to stop microwave radiation instantly, reducing sample overheating, and the opportunity to use low reaction temperatures, which makes microwave methods reproducible and simple. The elevated pressure that the reaction system can reach during microwave irradiation is the main disadvantage of this technique [21].

The hydrothermal method, generally performed in an autoclave, is nowadays very popular, because the process can be controlled easily. Usually, aqueous solutions with the appropriate precursors are heated directly. Various molecules were used as precursors for CQD, including citric acid and amino acids. The hydrothermal treatment of renewable resources, such as aloe, lychee peel, silk or pepper [22-24], was also proposed to obtain CQD in accordance with "green chemistry". Due to the high pressure and temperature, the reaction yield is generally high, but the control over CQD size is poor [25].

The functionalization procedures of CQD play a fundamental role not only in the PL modulation, but also in many other applications including ORR catalysis [26-28], selective oxidation of organic molecules [29], doping of semiconducting membranes [30], drug delivery [19, 23], chemical or biosensing $[4,31]$ etc. The functionalization can lead to bulk doping or to definite defect organizations, such as surface passivation by noncovalent bonds or by covalent linkages of different molecules in edge sites.

In this work, we investigated how the combination of three synthetic approaches, three starting materials, and five N-based modifiers could influence CQD properties. The aim was to find the best conditions to tailor the nature of CQD to achieve nitrogen doping, surface passivation by molecules with Van der Waals interactions, and strong covalently bonded structures.

The synthetic methods included the classical pyrolysis technique, the microwave irradiation route and the hydrothermal approach in an autoclave reactor, while the different green precursors were citric acid, glucose, and glucose amine hydrochloride. The N-based modifiers ranged from hydrophilic to hydrophobic molecules, such as aminoacids, diamine or octylamine. The tailored CQD were studied in terms of composition, microstructure, thermal stability, and photoluminescence.

\section{Experimental}

\section{Materials}

D-(+)-glucose $(\mathrm{G}), \mathrm{D}-(+)$-glucosamine hydrochloride (GA), citric acid (CA), 6-aminohexanoic acid (6AHA), $\mathrm{N}$-octylamine (Oct), N,N-dimethylamine (DMA), 1,6-diaminohexane (DAH), tryptophan (Try), and the other chemicals were reagent-grade and were used as received from Aldrich.

The following abbreviations are used in the text.

CAP: CQD from citric acid by pyrolysis.

CAPDMA: CQD from citric acid functionalized with dimethylamine.

CAPOct: CQD from citric acid functionalized with $\mathrm{N}$-octylamine.

GM: CQD from glucose by microwave irradiation.

GM6AHA: CQDs from glucose functionalized with 6-aminohexanoic acid.

GMTry: CQDs from glucose functionalized with L-tryptophan.

GAH: CQDs from glucosamine hydrochloride by hydrothermal synthesis.

GAHOct: CQDs from glucosamine hydrochloride functionalized with N-octylamine.

GAHDAH: CQDs from glucosamine hydrochloride functionalized with 1,6-diaminohexane.

\section{Synthesis}

\section{CQD from citric acid by pyrolysis}

Bare CQD (CAP) $2 \mathrm{~g}$ of citric acid ( $15 \mathrm{mmol})$ were inserted in a 3-neck flask equipped with a water condenser and treated in a heating mantle at $200{ }^{\circ} \mathrm{C}$ for $10 \mathrm{~min}$. During that time, citric acid melted and the colour of the liquid changed from colourless to yellow and finally to orange. The liquid was cooled to room temperature and its $\mathrm{pH}$ was adjusted at 9 adding dropwise a $1.5 \mathrm{M} \mathrm{NaOH}$ solution under stirring. The final aqueous solution was filtered $(0.2 \mu \mathrm{m}$ membrane filter $)$ and purified in $2 \mathrm{kDa}$ dialysis bag.

\section{CQD functionalized with dimethylamine (CAP-}

DMA) Using the same apparatus setup, CAPDMA were obtained cooling the orange liquid before solidification and adding DMA (2 $\mathrm{M}$ in THF, $7.5 \mathrm{~mL}$ ) under nitrogen flux (molar reagent ratio $\mathrm{CA}: \mathrm{DMA}=1: 1$ ). The mixture 
was kept under stirring and heated at $65^{\circ} \mathrm{C}$ for $24 \mathrm{~h}$, then $1.5 \mathrm{M} \mathrm{NaOH}$ solution was added dropwise to the viscous mixture at room temperature up to $\mathrm{pH}=9$. The final mixture was centrifuged $(9500 \mathrm{rpm}, 30 \mathrm{~min})$ and the solid dissolved in $19 \mathrm{~mL}$ of deionized water. This solution was purified as previously reported.

GQD functionalized with N-octylamine (CAPOct) Following the procedure used for CAPDMA, CQD functionalized with N-octylamine were obtained adding $5 \mathrm{~mL}$ of THF and $2.5 \mathrm{~mL}$ of $\mathrm{N}$-octylamine to the orange liquid (molar reagent ratio CA:Oct $=1: 1$ ). THF was added to reproduce the experimental condition used for CAPDMA. A sudden change of colour to dark violet was observed. After $24 \mathrm{~h}$ at $65^{\circ} \mathrm{C}$ the dark greenish THF solution was centrifuged $(9500 \mathrm{rpm}$, $40 \mathrm{~min}$ ) obtaining a sediment and a liquid part used for the characterization.

\section{CQD from glucose by microwave irradiation}

Bare CQD (GM) $1.0 \mathrm{~g}$ of glucose (5.5 mmol), $19 \mathrm{~mL}$ of deionized water, and $1 \mathrm{~mL}$ of $2 \mathrm{M} \mathrm{HCl}$ were put in a glass bottle. The mixture was stirred for about 10-15 min until a clear solution was obtained, then placed in a microwave oven at a power of $800 \mathrm{~W}$ for $4 \mathrm{~min}$. After this time, a black precipitate was observed; the glass bottle was cooled to room temperature and the precipitate dissolved under stirring in $10 \mathrm{~mL}$ of deionized $\mathrm{H}_{2} \mathrm{O}$ for $5-10 \mathrm{~min}$. The brown solution was centrifuged (9500 rpm, $30 \mathrm{~min}$ ) obtaining a sediment and a liquid part used for the characterization.

\section{CQD functionalized with 6-aminohexanoic acid (GM6AHA)}

$0.51 \mathrm{~g}$ of glucose $(2.8 \mathrm{mmol})$ and $0.37 \mathrm{~g}$ of 6-aminohexanoic acid $(2.8 \mathrm{mmol})$ were dissolved under stirring in $19 \mathrm{~mL}$ of $\mathrm{H}_{2} \mathrm{O}$ and $1 \mathrm{~mL}$ of $2 \mathrm{M} \mathrm{HCl}$ in a $500 \mathrm{~mL}$ glass bottle. After $30 \mathrm{~min}$, a clear solution was obtained and placed in a microwave oven following the above procedure. The formed black precipitate was dissolved in $10 \mathrm{~mL}$ of deionized $\mathrm{H}_{2} \mathrm{O}$ and the solution was stirred for about 5-10 min and then centrifuged. No sedimentation was observed. Elemental analysis (atom\%): O 33.4, C 47.4, N 5.2, and H 7.9.

CQDs functionalized with L-tryptophan (GMTry) The same procedure was carried out for GMTry. Glucose and L-tryptophan (2.5 mmol, ratio 1:1) dissolved in $19 \mathrm{~mL}$ of $\mathrm{H}_{2} \mathrm{O}$ and $1 \mathrm{~mL}$ of $2 \mathrm{M} \mathrm{HCl}$ were placed in a microwave oven at a power of $8000 \mathrm{~W}$ for $4 \mathrm{~min}$. The black precipitate was dispersed in water and centrifuged (9500 rpm, $40 \mathrm{~min}$ ). After the centrifugation, the sediment part was removed and the brown liquid portion centrifuged again and then used for the analysis.

\section{CQD from glucosamine hydrochloride by hydrothermal synthesis}

Bare CQD (GAH) $0.45 \mathrm{~g}$ of glucosamine hydrochloride $(2.1 \mathrm{mmol})$ was put in $1 \mathrm{~mL}$ of $\mathrm{H}_{2} \mathrm{O}$ and stirred for $40-50 \mathrm{~min}$ at $50{ }^{\circ} \mathrm{C}$ to obtain a solution. The solution was then transferred in an autoclave reactor and inserted into a preheated oven at $190{ }^{\circ} \mathrm{C}$ [32]. After $12 \mathrm{~h}$ the autoclave was cooled down to room temperature and a black soft solid was recovered and dispersed in deionized water. After centrifugation (9500 rpm, $40 \mathrm{~min}$ ) a small residue was separated and the solution was used for further characterization.

CQD functionalized with octylamine (GAHOct) $0.45 \mathrm{~g}$ of glucosamine hydrochloride $(2.1 \mathrm{mmol})$ and $0.27 \mathrm{~g}$ of N-octylamine were mixed in $1 \mathrm{~mL}$ of $\mathrm{H}_{2} \mathrm{O}$ and stirred for $1 \mathrm{~h}$ at $50{ }^{\circ} \mathrm{C}$. The solution was put in an autoclave as reported before. The formed black solid was insoluble in water; chloroform was used for the dispersion. The centrifugation (8500 rpm, $10 \mathrm{~min}$ ) afforded a liquid part, used for the characterization, and a small residue.

CQD functionalized with 1,6-diaminohexane (GAHDAH) The same procedure was carried out for the GAHDAH sample, using $0.45 \mathrm{~g}$ of GA and $0.24 \mathrm{~g}$ of 1,6-diaminohexane (molar ratio 1:1). After the autoclave reaction the solid was dispersed in methanol and then centrifuged (8500 rpm, $10 \mathrm{~min}$ ). No sedimentation was observed.

\section{Characterization}

\section{FTIR}

FTIR spectra were recorded in transmission mode in the range of $4000-400 \mathrm{~cm}^{-1}$ using a spectrometer Perkin-Elmer Spectrum 100. The dry samples were analysed in cesium iodide cells. Spectra were normalized against a background spectrum.

\section{NMR}

${ }^{13} \mathrm{C}$ NMR spectra were recorded with a Bruker AVANCE III spectrometer operating at $400 \mathrm{MHz}$. The samples were dried and then dissolved in the deuterated solvent (DMSO $\mathrm{d}_{6}, \mathrm{D}_{2} \mathrm{O}$, and toluene $\mathrm{d}_{8}$ ). Chemical shifts (ppm) were referenced to tetramethylsilane (TMS). 


\section{Transmission Electron Microscopy (TEM)}

TEM samples were prepared from a droplet dried in carbon film under infrared radiation for a maximum duration of $120 \mathrm{~s}$. The carbon film had a mean thickness of $50 \mathrm{~nm}$. All TEM inspections were carried out with a PhilipsTM CM-20® working at $200 \mathrm{kV}$ with a double-tilt specimen holder equipped with liquid nitrogen cooling stage. To acquire a sound statistical evaluation, a minimum of three samples of CAP and GM6AHA were inspected.

\section{X-ray photoelectron spectroscopy (XPS)}

The samples for X-ray photoemission spectroscopy analyses were prepared by depositing a drop of solution on grated $\mathrm{Au}$ foil (purity 99.99\%), then they were dried in air. Photoemission spectra were acquired using a spectrometer Escalab 250Xi (Thermo Fisher Scientific Ltd., East Grinstead, UK) with a monochromatic $\mathrm{Al} \mathrm{K \alpha}(1486.6 \mathrm{eV})$ excitation source and six-channeltron detection system. All the spectra were collected at a pass energy of $40 \mathrm{eV}$ in standard mode of electromagnetic lens system corresponding to an analysis area of approximately $1 \mathrm{~mm}$ in diameter. The binding energy (BE) scale was corrected for a low static charging by positioning the $\mathrm{C} 1 \mathrm{~s}$ peak of aliphatic carbon $(\mathrm{C}-\mathrm{C}, \mathrm{C}-\mathrm{H}$ bonds) at $\mathrm{BE}=285.0 \mathrm{eV}$ and controlling if the Fermi level corresponds to $\mathrm{BE}=0 \mathrm{eV}$. Spectroscopic data were processed by the Avantage v.5 software (Thermo Fisher Scientific Ltd).

\section{Thermogravimetric analysis (TGA)}

The thermograms were recorded using a high resolution thermogravimetric analyser TA Q500. The experiments were conducted with Pt sample holders, a CQD mass of about $7 \mathrm{mg}$, a temperature range $50-700{ }^{\circ} \mathrm{C}$ and a nominal heating rate of $3 \mathrm{~K} / \mathrm{min}$ under air flux.

\section{Optical measurements}

All the measurements for optical characterization were performed by keeping the solutions of CQD in fused-silica cuvettes with an optical path-length of $10 \mathrm{~mm}$. UV-Vis absorption spectra were recorded using a Varian Cary 50 Scan spectrophotometer. Photoluminescence (PL) emission spectra were acquired with a specific laboratory setup based on a continuous-wave 200-W $\mathrm{Hg}(\mathrm{Xe})$ discharge lamp (Oriel Corp.), an excitation 25-cm monochromator (PMI), an emission 25-cm monochromator (Oriel Cornerstone 260) and a Hamamatsu R3896 photomultiplier for detection of the emission light. Appropriate optical filters and the conventional $90^{\circ}$ geometry were used for improved stray-light rejection and signal-to-noise ratio. PL measurements were performed in diluted solutions in spectroscopic grade solvents, with an absorbance $<0.1$ optical density (OD) in the whole excitation/emission range. The typical excitation wavelength of $\lambda_{\text {exc }}=355 \mathrm{~nm}$ was used with a spectral bandpass of $3 \mathrm{~nm}$ for both the excitation and emission monochromators. The spectra were corrected for the instrumental response through a calibration performed using a reference black-body lamp and standard-fluorophore solutions. The relative quantum yield of the fluorescent emission was determined using both quinine sulphate and 9,10-Diphenylanthracene as reference and solutions with an absorbance $<0.05$ OD in the whole excitation/emission range.

\section{Results and discussion}

Scheme 1 shows the paths followed for the synthesis of bare and functionalized CQD. The structure of the products proposed in the Scheme is in agreement with the results obtained from the different analysis. The synthesis by pyrolysis is reported to start with the thermal decomposition of CA [33]. CA dehydrates around $175^{\circ} \mathrm{C}$ with the formation of aconitic acid, the decomposition continues until $225^{\circ} \mathrm{C}$ reaching $80 \%$ of weight loss around $200{ }^{\circ} \mathrm{C}$. This temperature, selected in the current work, affords small particles, around $1.4 \mathrm{~nm}$ diameter, although the PL, after a treatment of $10 \mathrm{~min}$, is relatively low [34]. The time of $10 \mathrm{~min}$ at $200^{\circ}$ $\mathrm{C}$ was chosen to have a balance between the hydroxyl and carboxyl groups, used for the subsequent functionalization, and the graphitic part of CQD. A longer time would lead to an increase in carbon-carbon bonds to the detriment of the oxygenated groups [34]. The formation of CQD is considered to take place via the synthesis of aromatic clusters by aldol condensation and cycloaddition, reactions catalysed typically in basic media. The balance of $\mathrm{pH}$ can be critical to achieve the aggregation reactions between carbon nanoparticles and an optimum value of $\mathrm{pH}=9$ was chosen for obtaining CQD with small size [3]. The bare CAP were postfunctionalized with $\mathrm{N}, \mathrm{N}$-dimethylamine and $\mathrm{N}$-octylamine. Due to the low reaction temperature $\left(65^{\circ} \mathrm{C}\right)$, the functionalization occurs via the formation of amide and amine bonds (see FTIR discussion) and nitrogen is not part of the skeletal network (Scheme 1a).

Both the synthesis of GM and GAH begin from carbohydrates. These starting materials are very attractive due to their accessibility, high solubility in water, and low carbonization temperatures [35]. The reaction starts by the opening of the anomeric carbon followed by polymerisations and aromatic or hetero-aromatic cluster formation [36, 37].

The microwave mediated glucose reaction is an acidcatalysed dehydration that produces alcohols and carboxylic acids on the CQD surface responsible of the solubility in polar solvents (Table 1). The surface passivation was obtained by reaction of glucose in the presence of amino 
a

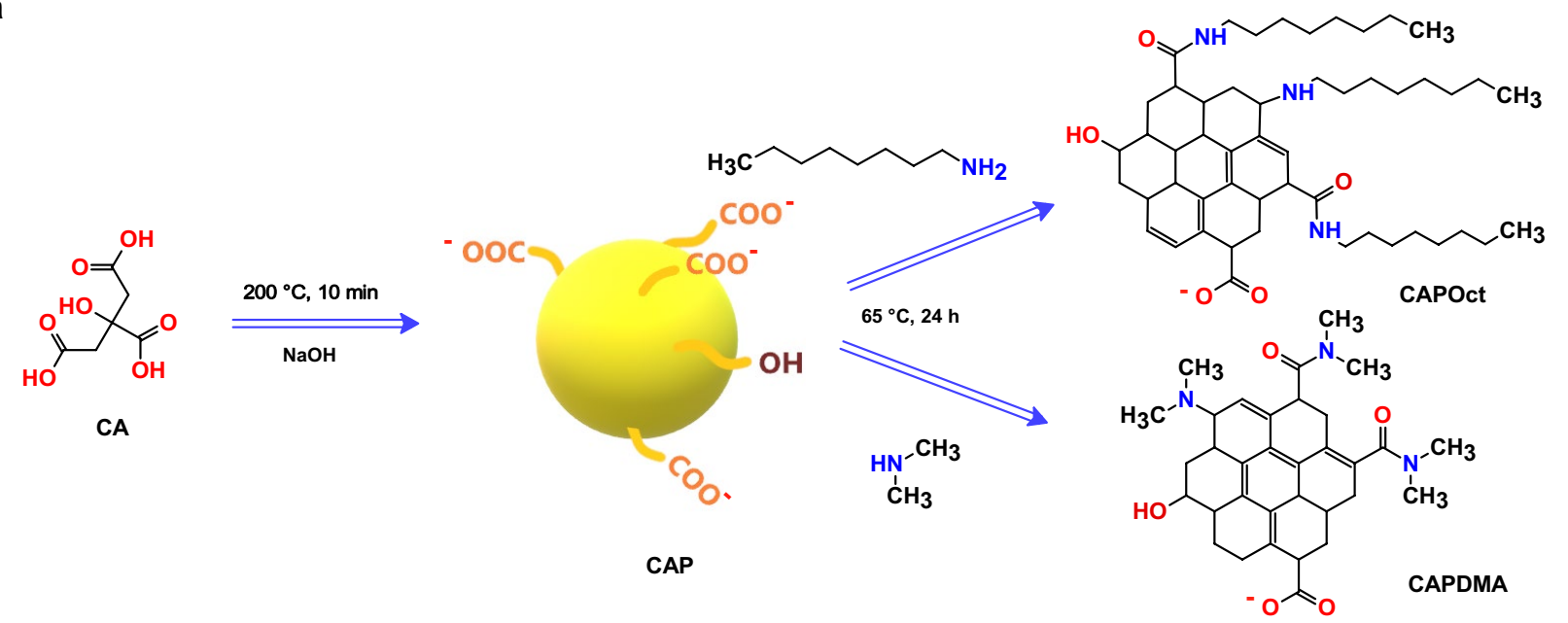

b

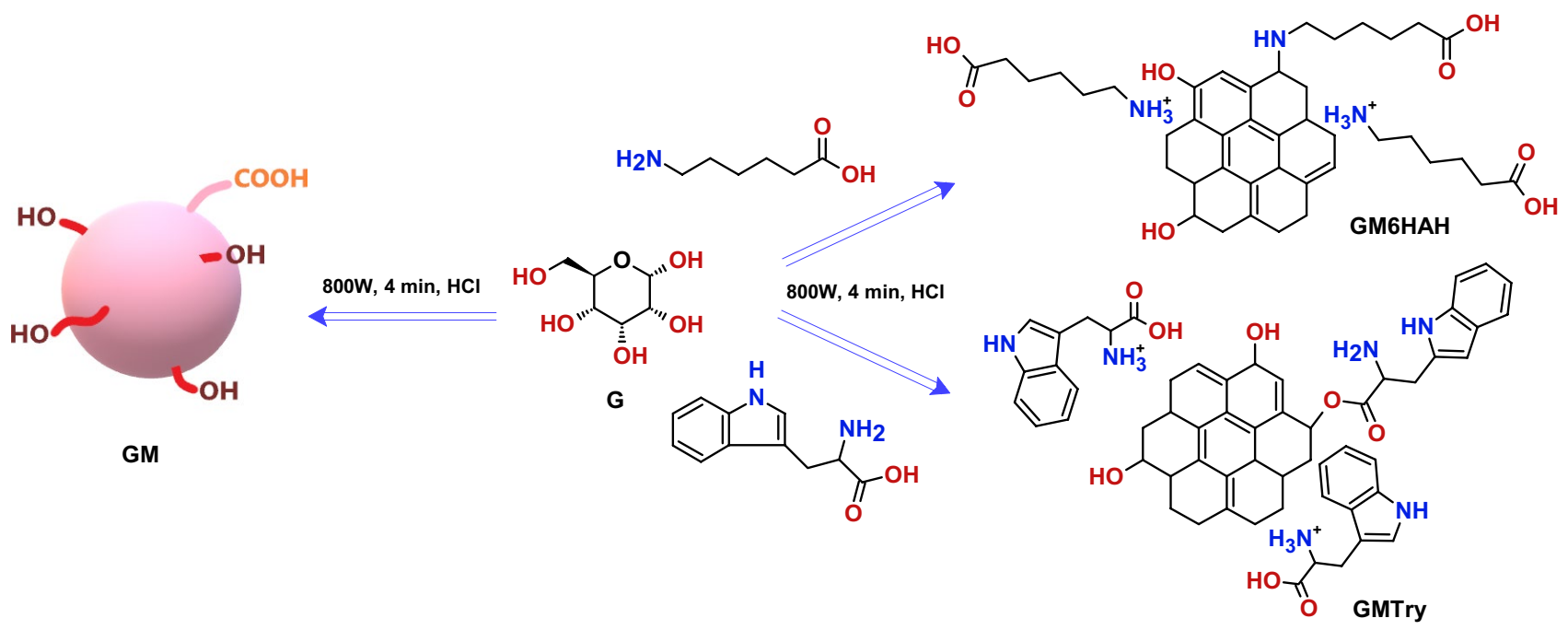

C

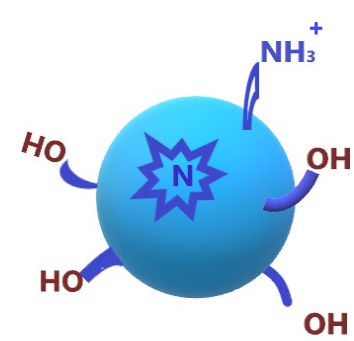

$\mathrm{OH}$

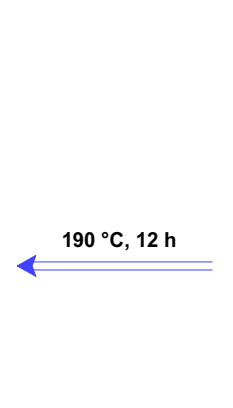

GAH

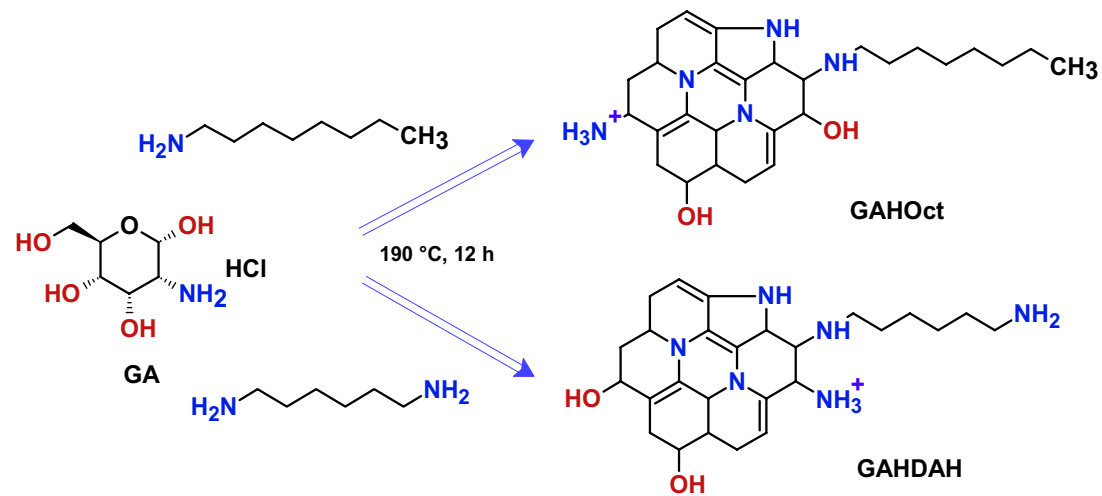

Scheme 1. Schematic synthesis routes for CQD made by (a) pyrolysis, (b) microwave irradiation, (c) hydrothermal treatment

acids. As reported in Scheme 1b, nitrogen is linked as amine to the CQD shell or present as passivating agent (see FTIR, NMR, and TEM). This behaviour was attributed to the weak interaction between glucose and functionalizing compounds by mostly van der Waals forces and hydrogen bonds [38]. It was also reported that CQD synthetized by microwave 
Table 1 Solubilities of CQD in various solvents. $+:>5 \mathrm{~g} / \mathrm{L}$

\begin{tabular}{|c|c|c|c|c|c|c|c|c|c|}
\hline $\begin{array}{l}\text { Solvent and } \\
\text { Dielectric con- } \\
\text { stant }(\varepsilon) \\
\text { Samples }\end{array}$ & $\begin{array}{l}\mathrm{H}_{2} \mathrm{O} \\
80\end{array}$ & $\begin{array}{l}\text { DMSO } \\
47\end{array}$ & $\begin{array}{l}\text { DMAc } \\
38\end{array}$ & $\begin{array}{l}\text { DMF } \\
37\end{array}$ & $\begin{array}{l}\mathrm{MeOH} \\
33\end{array}$ & $\begin{array}{l}\text { NMP } \\
32\end{array}$ & $\begin{array}{l}\text { THF } \\
8\end{array}$ & $\begin{array}{l}\mathrm{CHCl}_{3} \\
5\end{array}$ & $\begin{array}{l}\text { Toluene } \\
2\end{array}$ \\
\hline CAP & + & + & - & + & + & - & - & + & - \\
\hline CAPDMA & + & + & - & + & - & - & - & + & - \\
\hline CAPOct & - & - & + & + & - & + & + & + & + \\
\hline GM & + & + & + & + & - & + & - & - & - \\
\hline GMTry & + & + & + & + & + & + & + & - & - \\
\hline GM6AHA & + & + & + & + & + & + & - & - & - \\
\hline GAHOct & - & + & + & + & + & + & - & + & + \\
\hline GAHDAH & + & + & + & + & + & + & - & - & - \\
\hline
\end{tabular}

irradiation in the presence of water present a self-passivated layer on the surface [39].

CQD prepared from glucosamine hydrochloride by hydrothermal synthesis offer the advantage to contain nitrogen in the starting material. The reported mechanism includes the oligomerization of glucosamine molecules by intermolecular dehydration followed by polymerization and aromatization via intramolecular dehydration [40]. The functionalization was realized using 1,6-diaminohexane and N-octylamine and resulted in an increase of graphitic nitrogen.

The colloidal solubilities reported in Table 1 show that only CQD functionalized with octyl groups are insoluble in water, due to the hydrophobic nature of the long aliphatic chain. In fact, the CQD-Oct are the only samples soluble in toluene confirming the lyophilic nature. The CQD synthetized by microwave irradiation are instead insoluble in solvents with low dielectric constant and show a larger hydrophilicity, in agreement with the core-shell structure evidenced by TEM and XPS (see below). CQD by pyrolysis present a variable solubility without a clear trend in function of the dielectric constant indicating a more complicated substituent distribution.

Figure 1 illustrates the FTIR analysis of functionalized samples. FTIR spectra of bare samples and GMTry are reported in Supplementary Information (SI 1).

$\mathrm{OH}$ stretching is observed for all compounds around $3450 \mathrm{~cm}^{-1}$. The intensity is lower for the octylamine-functionalized CQD (Fig. 1a: CAPOct and Fig. 1b: GAHOct) in agreement with the hydrophobic nature of the long aliphatic chain. The C-H stretch of alkyl groups at $2960 \mathrm{~cm}^{-1}$ is evident for all compounds, but less pronounced for CAPDMA due to the absence of a long chain existing in all other samples. The $\mathrm{CO}$ stretching of carboxylic acid, originating from CA precursor, is present for CAPDMA at $1750 \mathrm{~cm}^{-1}$ and less evident for CAPOct. The amide CO vibration $\left(1680 \mathrm{~cm}^{-1}\right)$, showing a reaction with the amine group of the functionalizing molecule, is strong for CAPOct, while it exists as shoulder in CAPDMA. Regarding GM and GAH samples,
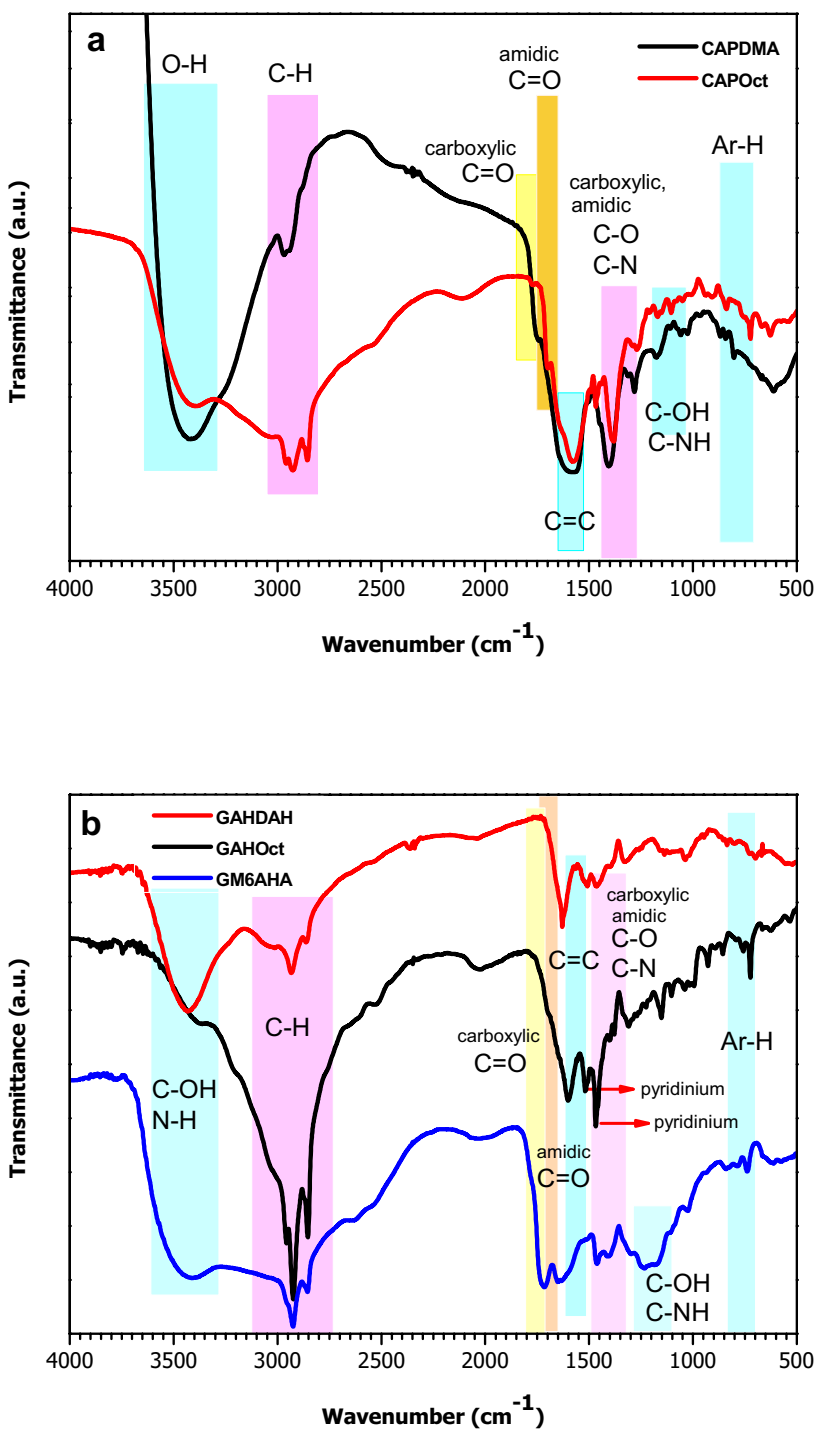

Fig. 1 Comparison of FTIR spectra for CQD made by: a pyrolysis (CAPDMA and CAPOct); b microwave irradiation (GM6AHA) and hydrothermal synthesis (GAHOct and GAHDAH) 
the $\mathrm{CO}$ stretching of carboxylic acid is at $1750 \mathrm{~cm}^{-1}$ for the bifunctional GM6AHA, while the $\mathrm{CO}$ amide vibration is present as a shoulder for GAHDAH and GAHOct in the range 1660-1680 $\mathrm{cm}^{-1}$ and seems absent in GM6AHA sample because of the non-covalent core-shell structure. At $1640 \mathrm{~cm}^{-1}$, the bending absorption of $\mathrm{N}-\mathrm{H}$ is marked for GAHDAH, due to the two amine groups of its functionalized molecule; this band is less evident for GAH6AHA. The aromatic $\mathrm{C}=\mathrm{C}$ vibration around $1610 \mathrm{~cm}^{-1}$ is observed especially for CAPDMA, CAPOct, and GAHOct, less intense for GM6AHA and GAHDAH. The peak at $1530 \mathrm{~cm}^{-1}$, strong for GAHOct and weak for GAHDAH, can be assigned to the bending vibration of pyridinium ions [41]; this peak is associated with the bending of pyridine around $1450 \mathrm{~cm}^{-1}$, visible in both samples of Fig. 1b, and as shoulder in CAPDMA (Fig. 1a). CAPDMA and GM6AHA show an absorption at $1400 \mathrm{~cm}^{-1}$ characteristic of $\mathrm{C}-\mathrm{O}$ vibrations of carboxylic acid and [42]. In the other samples, this signal is less pronounced or shifted (CAPOct, $1360 \mathrm{~cm}^{-1}$ ) indicating the transformation of carboxylic acid into amide, in agreement with the previous consideration. A band at $1315 \mathrm{~cm}^{-1}$ is observed for GAHOct and can be assigned to graphitic nitrogen, in agreement with XPS results [43]. At $1150 \mathrm{~cm}^{-1}$ the $\mathrm{C}-\mathrm{N}$ stretching is observed especially for GAHOct, GADAH, and CAPDMA. In the range $700-760 \mathrm{~cm}^{-1}$ aromatic $\mathrm{C}-\mathrm{H}$-bending vibrations are visible. A summary of peak intensities and assignments is reported in SI 2.

${ }^{13} \mathrm{C}$ NMR spectra of various samples are reported in Fig. 2. Three main regions are roughly identified in the spectrum of Fig. 2a. The $\mathrm{sp}^{3}$ zone can be further subdivided in high-field and low-field regions, where alkylic and $\mathrm{C}-\mathrm{X}$ $(\mathrm{X}=\mathrm{O}, \mathrm{N})$ carbons absorb.

The CAPDMA spectrum shows that the nitrogen of the DMA moiety is connected to CQD by a direct linkage $\left(\mathrm{N}-\underline{\mathrm{CH}}_{3}\right.$ and $\underline{\mathrm{C}}-\mathrm{N}$ peaks around 45 and $\left.55 \mathrm{ppm}\right)$ and an amidic bond $\left(\underline{\mathrm{C}} \mathrm{H}_{3}-\mathrm{N}-\mathrm{CO} 35-38 \mathrm{ppm}\right.$ and $\mathrm{N}-\underline{\mathrm{C}}=\mathrm{O}$ around $170 \mathrm{ppm}$ ) as represented in Scheme 1a. In the region of $\mathrm{sp}^{2}$ carbons, the peaks between 120 and 130 ppm are ascribed to graphitic $\mathrm{C}$, such as polycyclic aromatic carbons, while peaks from 130 to $140 \mathrm{ppm}$ can be assigned to internal $\mathrm{C}=\mathrm{C}$ bonds. The existence of peaks between 110 and $120 \mathrm{ppm}$ was attributed to the presence of terminal $\mathrm{C}=\mathrm{C}$ bonds [44-46].

Peaks of amide moieties can be recognized around $170 \mathrm{ppm}$ and around $180 \mathrm{ppm}$. The CAPOct spectrum (Fig. 2b) does not show substantial changes from the previous one, except in the aliphatic region, where octylamine absorbs. For this sample, it is possible to conclude that the amine is linked to CQDs by aminic and amidic linkages, in agreement with the FTIR results. The spectrum is recorded in toluene to favour a good solubility of this hydrophobic sample, although the solvent obscures the $\mathrm{sp}^{2}$ region. More complex is the GM6AHA spectrum
(Fig. 2c) especially in the range $40-70 \mathrm{ppm}$, where there are the absorbances of $\mathrm{C}-\mathrm{O}$ and $\mathrm{C}-\mathrm{N}$ carbons, indicating a considerable amount of alcoholic and aminic groups also deriving from the functionalizing molecule. The peak at $95 \mathrm{ppm}$ can be ascribed to isolated double bonds in enolate form, and the absorbance at $150 \mathrm{ppm}$ to phenol or aniline moieties. This hypothesis is strengthened by the low quantity, or absence, of the amidic peaks around $170 \mathrm{ppm}$, confirming the FTIR observations. Figure $2 d$ shows the NMR spectrum of GMTry. In the range 108-137 ppm, it is possible to recognize the absorbance of tryptophan that maintains its structure after the synthesis of CQD. These peaks obscure the absorption of graphitic and polycyclic carbons. The tryptophan original signals at 56 and 175 ppm ( $\alpha$ carbon and carboxylic acid bearing in $\alpha$ an amino group, respectively) appear shifted in the spectrum (Fig. 2d) suggesting that a linkage with CQD occurs via ester and/or amino bonds.

The spectra of CQDs synthetized by the hydrothermal method present a different pattern and no carboxylic groups are present in Fig. 2e, f. Peaks around 150-160 ppm in GAHDAH (Fig. 2e) can be assigned to graphitic nitrogen-carbon bonds together with peaks at $140 \mathrm{ppm}$ indicating graphitic carbons. Signals around $120 \mathrm{ppm}$ can be attributed to pyrrolic groups, while in the range 60-70 ppm, we can recognize $\mathrm{C}-\mathrm{O}$ and $\mathrm{C}-\mathrm{N}$ peaks. Solubility issues affect the quality of the last spectrum, reported in Fig. 2f. The aliphatic part is very similar to that reported in Fig. 2b. A very small peak can be detected in the region 140-150 ppm, corresponding to $\mathrm{C}=\mathrm{C}$ bonds.

The TEM micrographs of CAP (Fig. 3a) and GAH (Fig. 3b) show bare CQD with a size distribution around an average of about $4 \mathrm{~nm}$, in good agreement with the literature [47]. Instead, the CQD functionalized with 6-aminohexanoic acid (GM6AHA, Fig. 3c, d) presents a core-shell structure. The shell width of around $0.9 \mathrm{~nm}$ is in perfect agreement with the six-carbon chain molecule. The core size distribution around an average value of $2.4 \mathrm{~nm}$ corresponds to around 16 carbon atoms in each direction, which is equivalent to about 6 six-membered rings or around 200 carbon atoms in the CQD core. Similar core-shell structures, confirmed by SAXS patterns, were reported recently with amino-acid-like molecules. The shell around CQD appears to be a weakly bonded reaction intermediate that collapses in later reaction stages or with higher energy methods, such as pyrolysis and hydrothermal synthesis [48].

Figure 4 shows XPS spectra of the $\mathrm{N} 1 \mathrm{~s}$ region including the peak fitting for the investigated samples and the survey spectrum for GMTry. Table 2 presents the BE values in $\mathrm{eV}$ and atomic concentrations of different nitrogen species. The spectra of the C $1 \mathrm{~s}$ region for the samples GAHOct, GAH, GMTry, and CAP are reported in the SI section (SI 3-7) with survey spectra and the attributions of carbon species. 
a CAPDMA
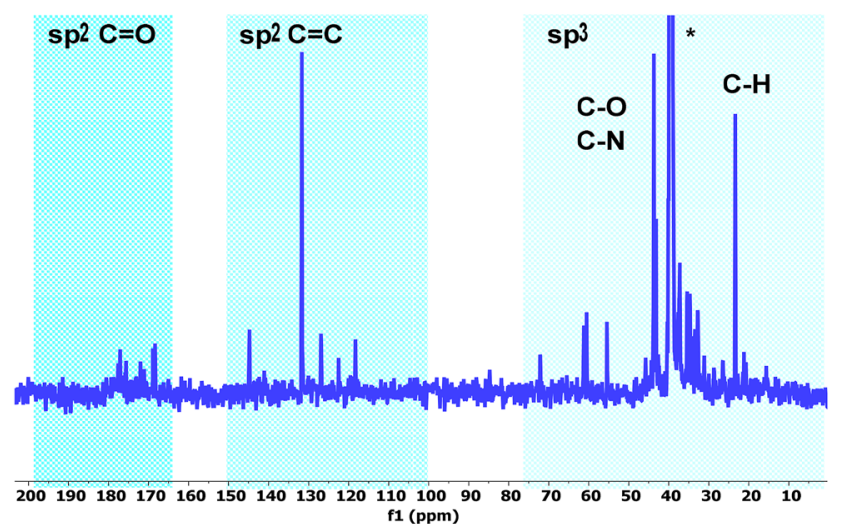

c GM6AHA

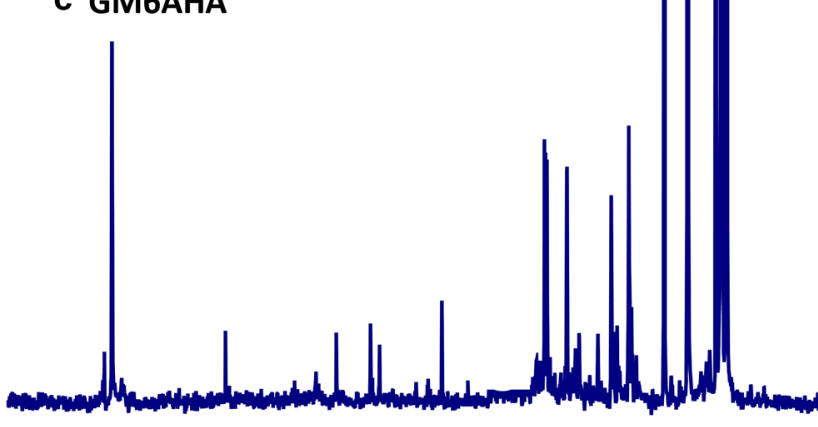

$200190180170160150140130120 \begin{gathered}110 \\ f 1(p p m)\end{gathered}$

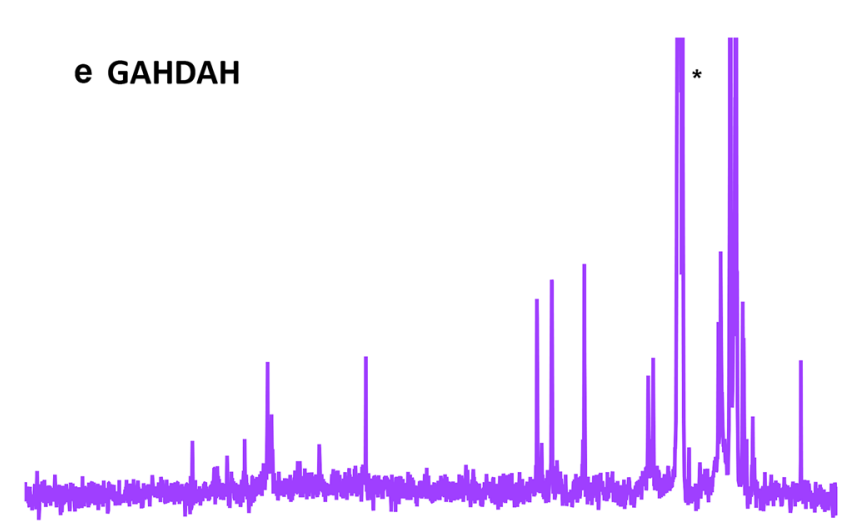

$\begin{array}{llllllllllllllllllllll}200 & 190 & 180 & 170 & 160 & 150 & 140 & 130 & 120 & 110 & 100 & 90 & 80 & 70 & 60 & 50 & 40 & 30 & 20 & 10\end{array}$

\section{b CAPOct}

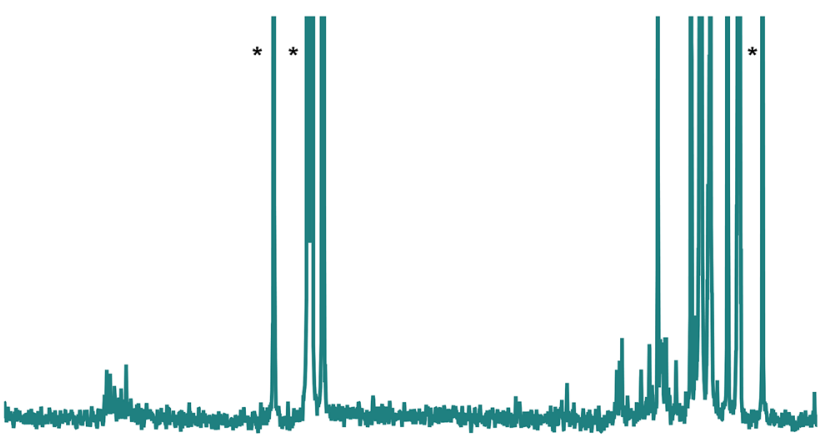

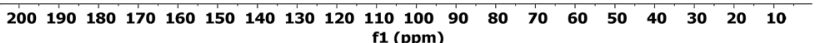

d GMTry

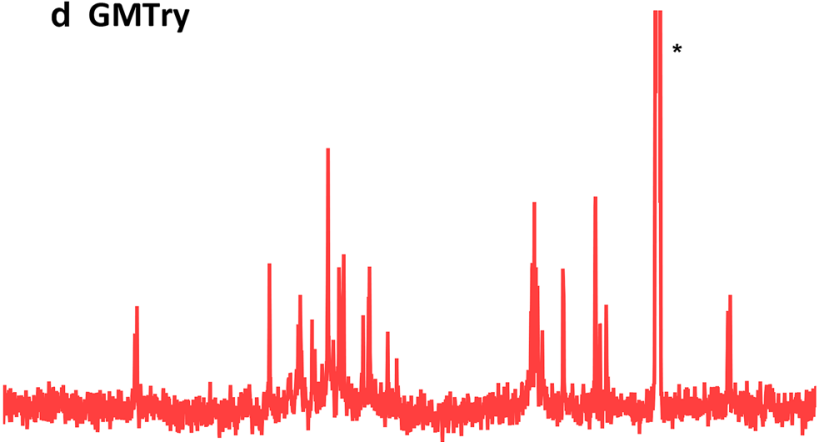

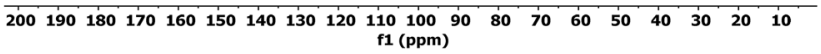

f GAHOct

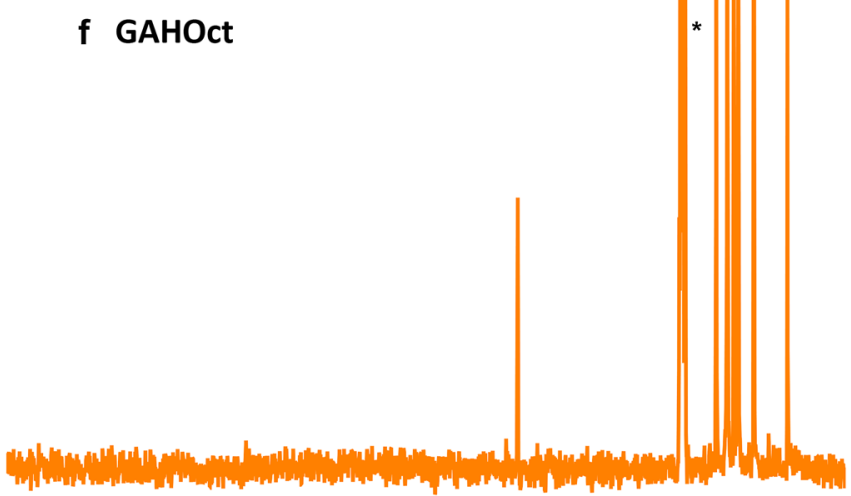

\begin{tabular}{llllllllllllllllllll}
\hline 200 & 190 & 180 & 170 & 160 & 150 & 140 & 130 & 120 & $\begin{array}{c}110 \\
f 1(p p m)\end{array}$ & 100 & 80 & 70 & 60 & 50 & 40 & 30 & 20 & 10
\end{tabular}

Fig. 2. ${ }^{13} \mathrm{C}$ NMR spectra of: (a) CAPDMA (DMSO d 6 ); (b) CAPOct (toluene $\mathrm{d}_{8}$ ); (c) GM6AHA $\left(\mathrm{D}_{2} \mathrm{O}\right)$; (d) GMTry (DMSO d $)$; (e) GAHDAH $\left(\right.$ DMSO d $_{6}$; (f) GAHOct (DMSO d 6 )

As described in ref. [43], it is possible to identify the chemical state of nitrogen in the CQD structure from XPS results: the $\mathrm{N} 1 \mathrm{~s}$ peaks for graphitic, pyrrolic, and pyridinic nitrogen in N-doped graphene are at BE of 401.5, 399.7, and $397.9 \mathrm{eV}$, respectively, whereas the hydrogenation of pyridinic $\mathrm{N}$ shifts its peak to $400.5 \mathrm{eV}$. Slightly different values for the $\mathrm{N} 1 \mathrm{~s}$ peak have been reported in ref. [7]: graphitic $\mathrm{N}$ at $401.3 \mathrm{eV}$, pyrrolic $\mathrm{N}$ at $400.2 \mathrm{eV}$ and pyridinic $\mathrm{N}$ at
$398.5 \mathrm{eV}$. More attributions of N $1 \mathrm{~s}$ peaks can be found in the refs. [49-52].

Pyrrolic nitrogen $(\mathrm{BE} \approx 400 \mathrm{eV})$ is observed in all the samples. The XPS results also indicate that during the hydrothermal synthesis some nitrogen is inserted in graphitic position $(\mathrm{BE} \approx 401 \mathrm{eV}$ ), especially when octylamine is present. The pyridinic nitrogen $(\mathrm{BE} \approx 398 \mathrm{eV})$ is observed only in the GAH sample that was prepared without functionalizing 
a

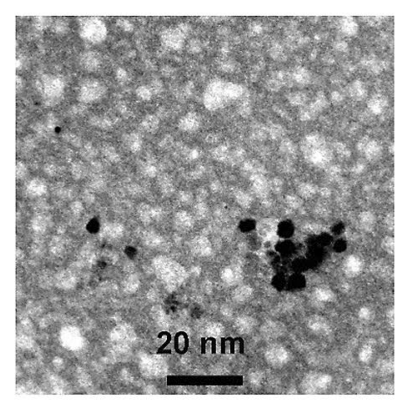

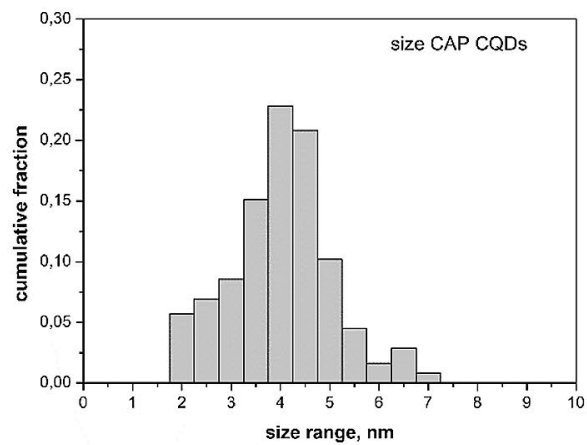

b
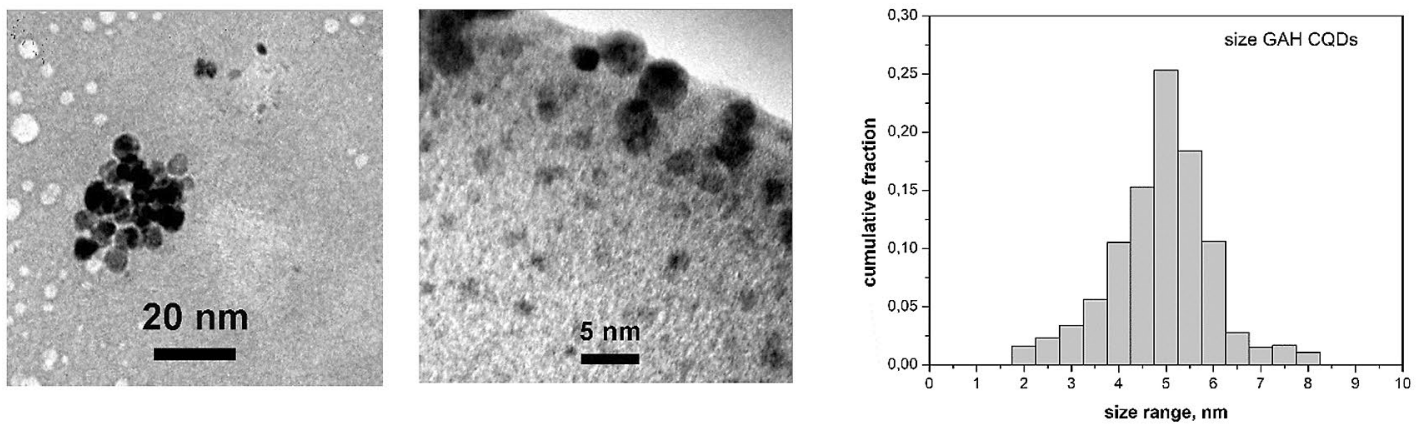

C
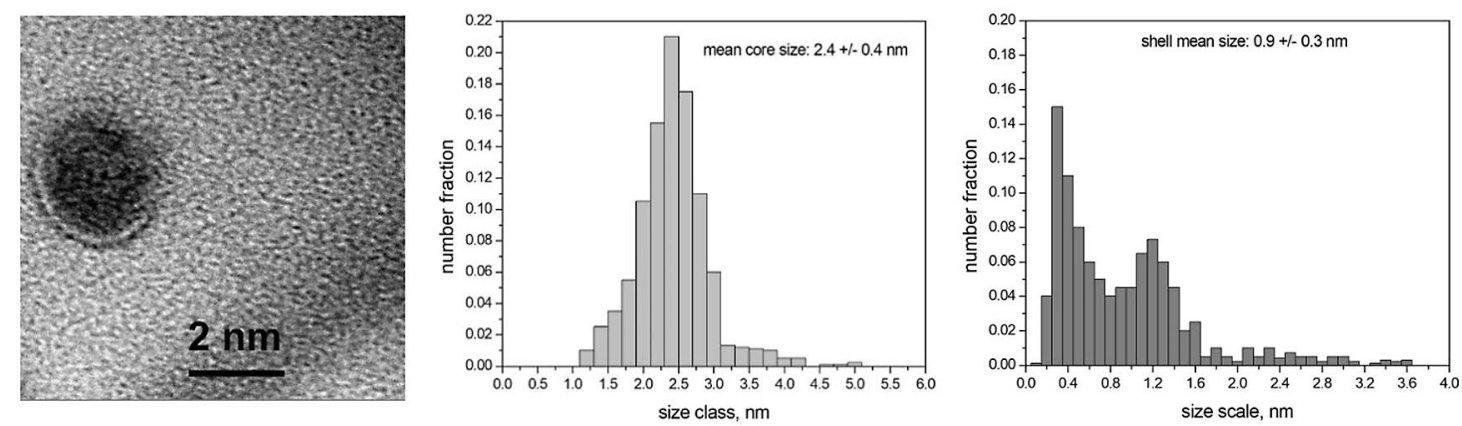

d
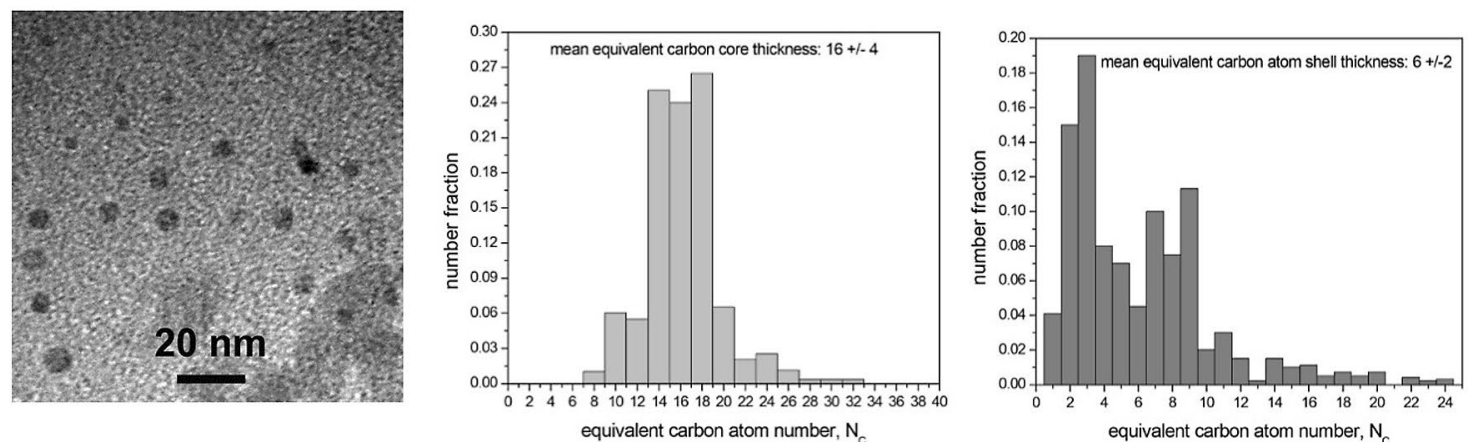

Fig. 3 BF-TEM micrographs and CQD size distribution for (a) CAP, (b) GAH and (c), (d) GM6AHA 

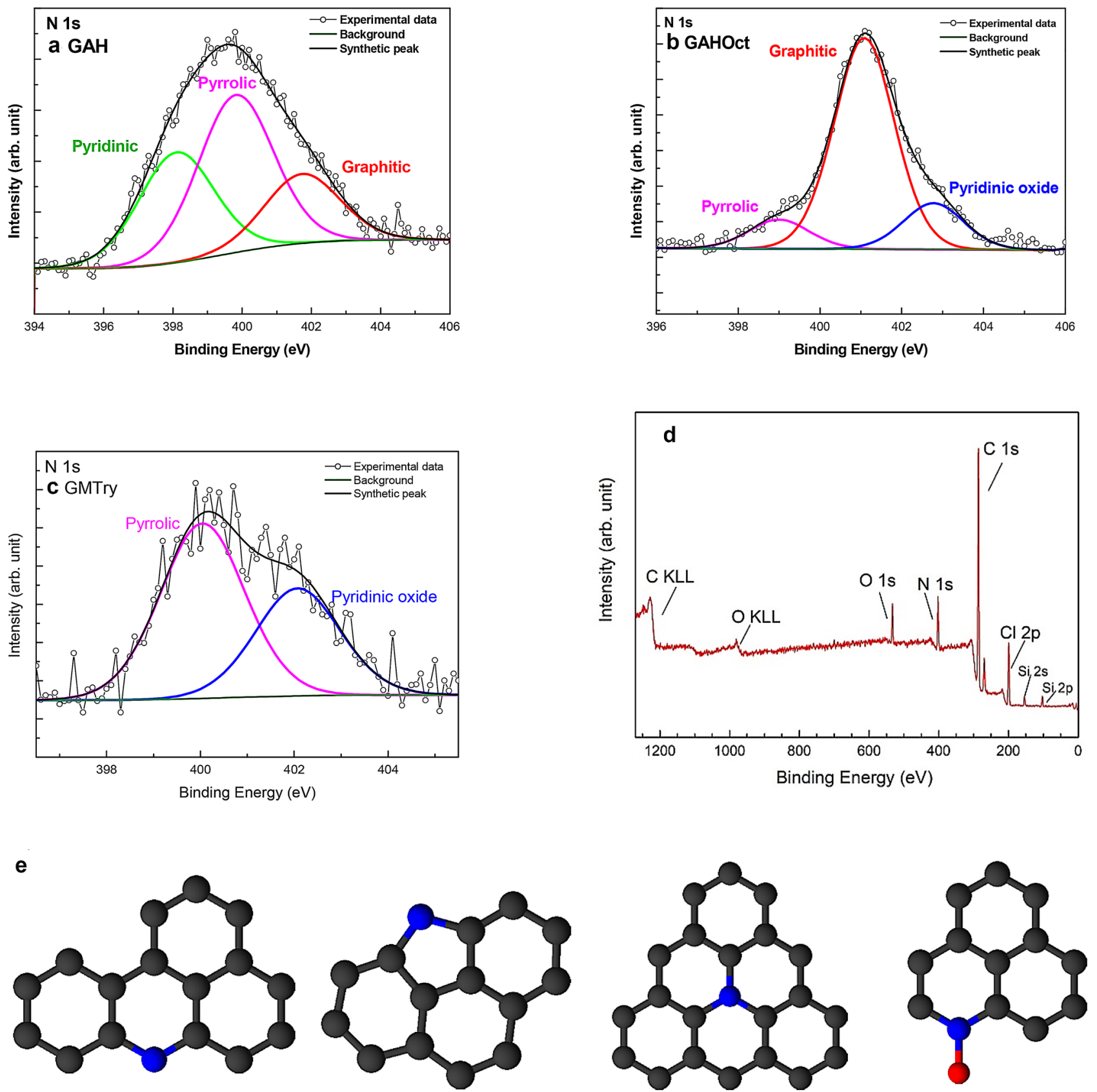

Fig. 4 XPS spectra of the N $1 \mathrm{~s}$ region for three samples: a) GAH, b) GAHOct c) GMTry; d) Survey spectrum of GMTry. The Si signals originate from the sample holder; e) Schematic representation of vari-

ous nitrogen bond types (from the left: pyridinic, pyrrolic, graphitic, pyridinic oxide)

Table 2 BE values and atomic concentrations of various nitrogen species in CQD

\begin{tabular}{lllll}
\hline $\begin{array}{l}\text { Nitrogen species } \\
\text { Sample }\end{array}$ & Pyridinic & Pyrrolic/Amine/Amide & Graphitic & Pyridinic oxide \\
\hline GAH & $398.0(2.5$ at $\%)$ & $399.7(3.7$ at $\%)$ & $401.6(1.6$ at $\%)$ & - \\
GAHOct & - & $399.0(1$ at $\%)$ & $401.1(7.4$ at $\%)$ & $402.8(1.6$ at $\%)$ \\
GMTry & - & $400.1(3.1$ at $\%)$ & - & $402.0(2.3$ at $\%)$ \\
\hline
\end{tabular}


molecule in hydrothermal conditions, whereas the pyridinic oxide species $(\mathrm{BE} \approx 402 \mathrm{eV})$ are only visible, when functionalizing molecules were added. The co-existence of pyrrolic and pyridinic oxide species and the absence of graphitic nitrogen in the sample GMTry are consistent with the presence of the functionalized molecules in a shell around the CQD core, such as in the case of GM6AHA observed by TEM. Both samples made by microwave synthesis present thus a core-shell structure, which is related to the lower energy provided by this method.

Figure 5 shows thermogravimetric curves (mass loss and derivative) for two samples made by hydrothermal synthesis and microwave irradiation. The peaks in the derivative curves correspond to the various thermal degradation processes. A first mass loss with a maximum around $100{ }^{\circ} \mathrm{C}$ is related to the loss of chemisorbed water. [29, 53] It is much more pronounced in the case of GM6AHA due to the hydrophilic nature of the carboxylic acid groups of 6-aminohexanoic acid, which strongly increases the hydrophilicity of this sample that is water soluble. This observation is in agreement with the core-shell structure of GM6AHA evidenced by TEM indicating that the functionalizing molecules are intact. Only a small water loss is noticed in the sample made by hydrothermal synthesis, because most of the diaminohexane has been transformed into structural nitrogen in the CQD. A small peak at $75{ }^{\circ} \mathrm{C}$ is probably related to physisorbed water on less hydrophilic amine and oxygenated groups, present in both samples. The broad mass loss between 150 and $400{ }^{\circ} \mathrm{C}$ in GAHDAH and GM6AHA is typical of CQD and is generally attributed to the loss of
Fig. 5 Thermogravimetric analysis of CQD from glucose functionalized with 6-aminohexanoic acid by microwave irradiation (GM6AHA) and from glucosamine hydrochloride functionalized with 1,6-diaminohexane by hydrothermal synthesis (GAHDAH): a mass loss curve, $\mathbf{b}$ derivative curve a

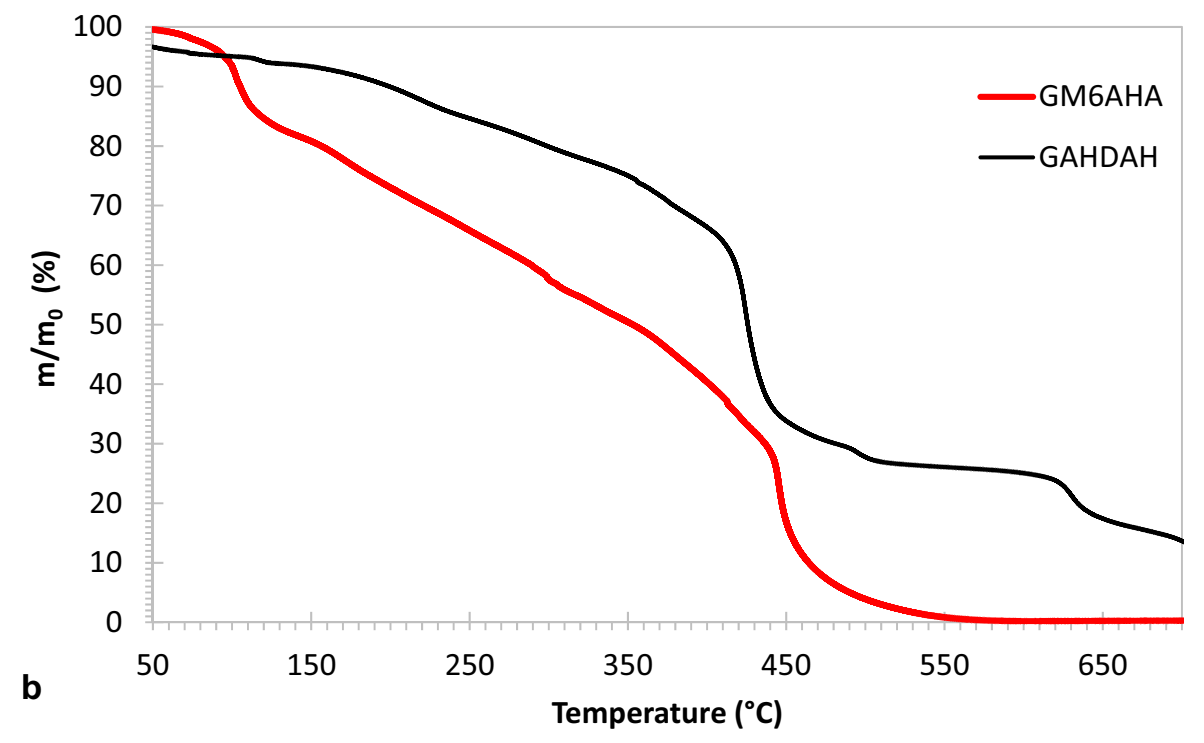


oxygenated groups, including hydroxyl, ketone, carboxylic acid, and amide [54]. The larger mass loss for GM6AHA indicates a larger amount of oxygenated groups [29] in agreement with elemental analysis, NMR, and XPS. The main decomposition process of CQD is observed with a peak maximum at 425 (GAHDAH) and $450{ }^{\circ} \mathrm{C}$ (GM6AHA), corresponding to the high stability of aromatic bonds in CQD.

The presence of the core-shell structure seems to enhance the thermal stability of GM6AHA. Small peaks about $40 \mathrm{~K}$ below the main peak are observable in both samples and correspond probably to a minor amount of $\mathrm{sp}^{3}$ carbon, as observed previously in poly(2,6-dimethyl-1,4-phenylene oxide), where the structural methyl groups are degraded before the aromatic main chain [55]. One notices that the degradation of the core-shell GM6AHA is terminated at $550{ }^{\circ} \mathrm{C}$, whereas a plateau is observed in the case of GAHDAH. This plateau was previously attributed to pyridinic and pyrrolic nitrogen, which present a high thermal stability and decompose only around $625^{\circ} \mathrm{C}$ [54].

Figure 6 displays the UV-Vis absorption spectra of the reference compounds, as prepared by the different methods of synthesis and starting materials without any functionalization. The absorbance spectrum of CAP (black continuous line) shows the typical profile found in the literature for this type of bottom-up preparation $[17,56]$. The general steep decrease of absorbance with increasing wavelength is usually assigned to the tail of high-energy $\pi^{*}-\pi^{*}$ transitions of aromatic $\mathrm{C}=\mathrm{C}$ bonds $[17,56]$. Differently, a controversial attribution has been given to the smooth peak/shoulder at $340 \mathrm{~nm}$. In the first work on CA-derived CQDs [17], the peak was supposed to originate from optical transitions in quantum-size ordered clusters of $\mathrm{sp}^{2}$ carbon atoms confined within a defective $\mathrm{sp}^{3}$ matrix. More recently, also in the framework of the results reported in several types of CQDs

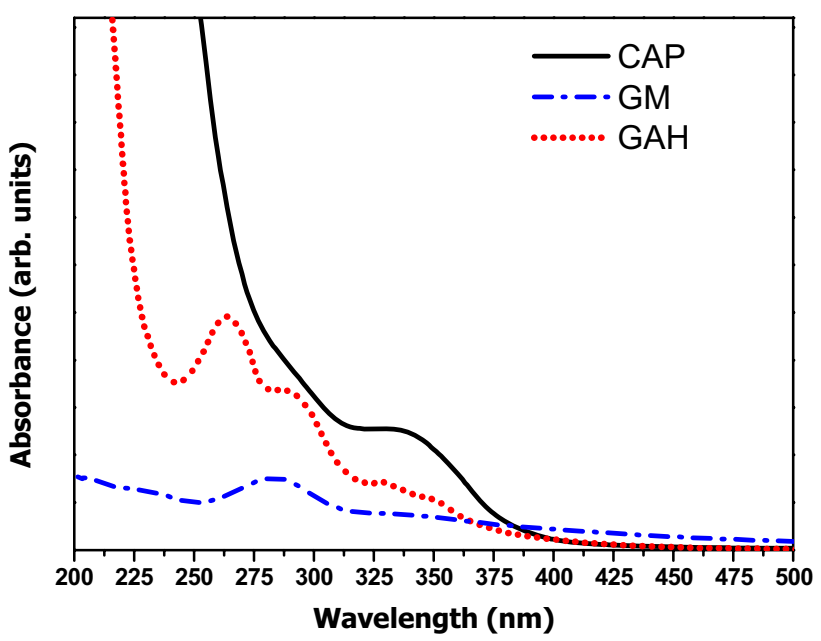

Fig. 6 UV-Vis absorption spectra of CQD made by: pyrolysis (CAP); microwave irradiation $(\mathrm{GM})$; hydrothermal treatment (GAH) prepared by top-down synthesis [57-59], similar structures were attributed to the $n-\pi^{*}$ transitions of surface states [56]. Surface states are synergistic hybridizations of the carbon backbone and linked oxygen-containing functional groups, such as carboxyl, hydroxyls and epoxy [57]. In the present case, a much smoother shoulder is also visible at $295 \mathrm{~nm}$ and is possibly originating from surface states, as well. In GM (blue dash/dot line), the structure at $340 \mathrm{~nm}$ is almost missing, while the shoulder at $295 \mathrm{~nm}$ has become a small yet clearly visible peak slightly shifted at $290 \mathrm{~nm}$, also found in previous reports on microwave-assisted synthesis [60]. Differently, when glucosamine and hydrothermal synthesis are used (GAH, red dotted line), the curve profile becomes more complex, with a distinct third peak at $270 \mathrm{~nm}$ that adds up to the smooth structures at $295 \mathrm{~nm}$ and $340 \mathrm{~nm}$.

The differences between pyrolysis and microwave syntheses in the relative intensities of the structures at 290 and $340 \mathrm{~nm}$ could originate from a diverse distribution and composition of the oxygen-containing functional groups. On the other hand, the structure at $270 \mathrm{~nm}$ arising in GAH is located at energies appreciably closer to those of $\pi^{*}-\pi^{*}$ transitions of aromatic $\mathrm{C}=\mathrm{C}$ bonds. This observation suggests that this peak can be assigned to new optical transitions due to nitrogen doping originating from the starting material and hydrothermal synthesis [56, 61]. In fact, the presence of graphitic and pyridinic nitrogen is confirmed by XPS data (see Table 2).

The UV-Vis absorption spectra of CAP derivatives are reported in SI 8. The data show that the intensity of the spectral structures at 290 and $340 \mathrm{~nm}$ progressively decreases with functionalization, especially in CAPOct. This can be explained by the nitrogen moieties substituting the oxygencontaining functional groups (Scheme 1), thus lowering the related absorbance [61].

Fig. SI 9 displays the UV-Vis absorption spectra of GM derivatives. In this series, functionalization with 6-aminohexanoic acid in GM6AHA only increases and slightly shifts the peak at $290 \mathrm{~nm}$ of the reference compound. Conversely, functionalization with tryptophan in GMTry produces a peculiar spectrum with a remarkable band at $280 \mathrm{~nm}$, very similar to the absorption feature of pure tryptophan itself, and a smaller peak at $360 \mathrm{~nm}$. Similar results have also been observed in functionalized CQD prepared with different methods [62], thus suggesting that this aminoacid retains its electronic level configuration when it is bound to CQD.

Finally, as regards the hydrothermal series (Fig. SI 10), functionalization with 1,6-diaminohexane in GAHDAH does not change the spectrum of the reference compound significantly. On the other hand, the curve of GAHOct is remarkably flat and does not present any appreciable structure, confirming the strong effect of octylamine on the functional groups, as observed in CAPOct, and its capacity to insert 


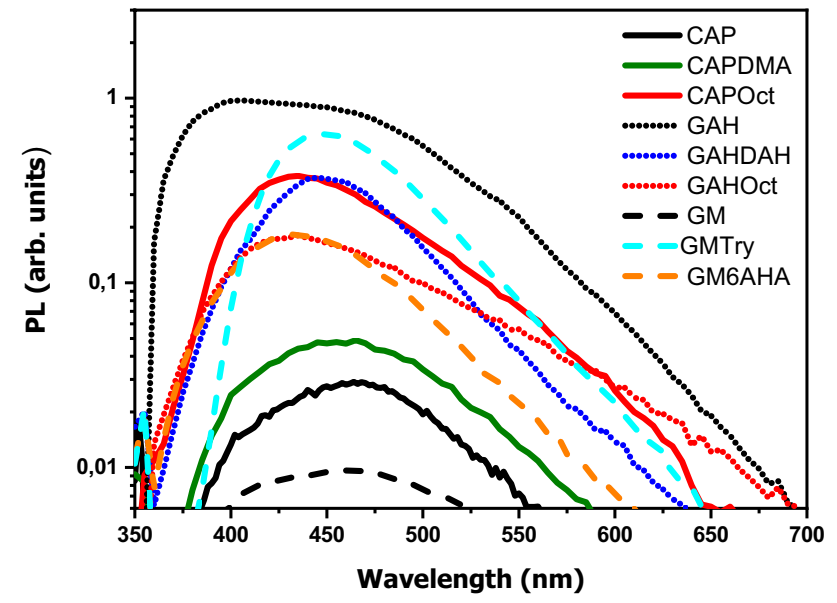

Fig. 7 PL emission spectra of CQD made by the different methods and various functionalization

nitrogen in graphitic position, as confirmed by XPS data (see Table 2).

In summary, if CAP are taken as reference compound/ method, the greatest and most significant changes of UV-Vis absorbance are produced using nitrogen-containing starting materials, especially octylamine, combined with hydrothermal synthesis. Smaller modifications are obtained with the less energetic microwave methods, except for functionalization with tryptophan that gives a material, which remarkably retains the absorption characteristics of the pure fluorophore along with a peculiar lower energy peak.

Figure 7 shows the photoluminescence (PL) emission spectra of the various CQD along with the respective derivatives and different functionalization. A semi-log plot has been used to due to large differences in the relative intensities and to display them more clearly. The excitation wavelength was $\lambda_{\text {ex }}=355 \mathrm{~nm}$ for all the samples. The spectra are normalized by the optical density at excitation wavelength, so that the plotted curves are representative of the relative fluorescence quantum yield.

As can be seen, all samples exhibit the blue fluorescence in the range around $450 \mathrm{~nm}$ which is typical of CA-derived CQD [17]. An appreciable short-wavelength contribution, which shifts the peak to $400 \mathrm{~nm}$, is only observed in GAH, while a smaller blue shift to $430 \mathrm{~nm}$ is produced by functionalization with octylamine and 6-aminohexanoic acid. However, it is evident that the functionalization does not alter the profile of emission spectra as much as it does for the absorption curves. This suggests that neither linked amino groups nor nitrogen doping of carbon backbone appreciably modify the recombination centers for excitons that are the surface defects due to non-perfect $\mathrm{sp}^{2}$ domains [56]. Instead, very different of PL signal can be observed, with variations well above one order of magnitude clearly indicating that functionalization affects the fluorescence quantum yield $\Phi$ of
Table 3 Quantum yield for the different CQD

\begin{tabular}{lc}
\hline Sample & $\Phi(\%)$ \\
\hline CAP & $0.6 \pm 0.2$ \\
CAPDMA & $1 \pm 0.2$ \\
CAPOct & $7 \pm 0.2$ \\
GAH & $24 \pm 2$ \\
GAHDAH & $6 \pm 0.2$ \\
GAHOct & $4 \pm 0.5$ \\
GM & $0.2 \pm 0.1$ \\
GMTry & $10 \pm 2$ \\
GM6AHA & $4 \pm 0.5$ \\
\hline
\end{tabular}

the emission process, as reported in Table 3. Especially, QY decreases in the order: GAH $>$ GMTry $>$ CAPOct $>$ GAH$\mathrm{DAH}>\mathrm{GM} 6 \mathrm{AHA} \cong \mathrm{GAHOct}>>\mathrm{CAPDMA}>\mathrm{CAP}>\mathrm{GM}$. We note that higher values of $Q Y$ are obtained when nitrogen-containing starting materials or functionalization are used. This is in agreement with the hypothesis that the introduction of nitrogen can produce new surface states that trap electrons and increase radiative recombination [56, 61]. In particular, the highest QY is observed in GAH that shows the presence of graphitic, pyrrolic and pyridinic nitrogen (see Table 2). Graphitic nitrogen can enhance the rigidity of the CQD structure thus reducing non-radiative relaxation through lattice vibrations [56].

Finally, Table 4 shows a comparison of the three synthesis methods.

\section{Conclusion}

We studied the synthesis of CQD starting from three "ecofriendly" molecules, citric acid, glucose and glucoseamine, by three different methods, pyrolysis, microwave irradiation, and hydrothermal synthesis. The CQD were functionalized using various nitrogen-containing compounds.

The investigation by various spectroscopic and thermal analysis methods and observation by transmission electron microscopy shows that the nature of CQD reflects the chemistry of the starting materials combined with the synthetic method.

The pyrolysis of citric acid leads to CQD rich of carboxylic moieties, very versatile groups that can be easily transformed into amide groups by reaction with amines. The nature of the functionalizing molecule influences the CQD hydrophilic/hydrophobic behaviour. The microwave irradiation of glucose gives materials with mainly alcoholic groups; the reaction in the presence of amino acids affords passivated CQD. This effect is probably related to the positive charge on the amino acids in acidic condition that allows strong electrostatic interactions. The hydrothermal treatment of glucosamine provides CQD with nitrogen in 


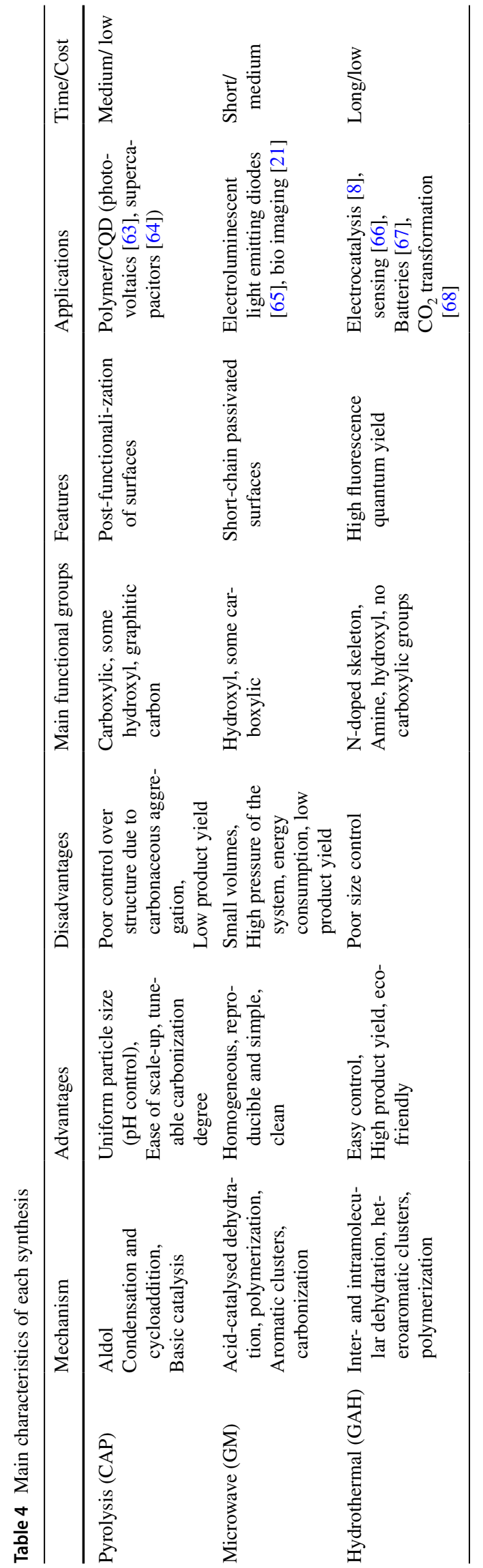

graphitic, pyrrolic and pyridinic positions. Further reactions with amines do not alter the CQD skeleton, but enhance the amount of nitrogen in the material.

The properties and the possible applications of CQD can thus be tuned: pyrolyzed CQD are functionalized by covalently bonded molecules and can be used as synthon compounds for example in the interaction with polymers. CQD obtained by the less energetic microwave process present passivation and formation of a core-shell structure with essentially Van der Waals bonds, very interesting for bio-applications. Finally, CQD by hydrothermal processes, the most energetic ones, exhibit bulk insertion of nitrogen that can act as a donor dopant. These samples show by far the highest quantum yields fundamental for sensing applications.

Supplementary Information The online version contains supplementary material available at https://doi.org/10.1007/s40097-021-00431-8.

Acknowledgements The research leading to these results received funding from the University of Rome "Tor Vergata", Italy for the project "Beyond Borders" (CUP: E84I20000260005).

Funding Open access funding provided by Università degli Studi di Roma Tor Vergata within the CRUI-CARE Agreement. The research leading to these results received funding from the University of Rome "Tor Vergata", Italy for the project "Beyond Borders" (CUP: E84I20000260005).

Availability of data and materials Not applicable.

Code availability Not applicable.

Declaration

Conflict of interest No conflict of interest.

Open Access This article is licensed under a Creative Commons Attribution 4.0 International License, which permits use, sharing, adaptation, distribution and reproduction in any medium or format, as long as you give appropriate credit to the original author(s) and the source, provide a link to the Creative Commons licence, and indicate if changes were made. The images or other third party material in this article are included in the article's Creative Commons licence, unless indicated otherwise in a credit line to the material. If material is not included in the article's Creative Commons licence and your intended use is not permitted by statutory regulation or exceeds the permitted use, you will need to obtain permission directly from the copyright holder. To view a copy of this licence, visit http://creativecommons.org/licenses/by/4.0/.

\section{References}

1. Dong, Y., Chen, C., Zheng, X., Gao, L., Cui, Z., Yang, H., Guo, C., Chi, Y., Li, C.M.: One-step and high yield simultaneous preparation of single- and multi-layer graphene quantum dots from CX-72 carbon black. J. Mater. Chem. 22, 8764-8766 (2012) 
2. Li, L., Wu, G., Yang, G., Peng, J., Zhao, J., Zhu, J.-J.: Focusing on luminescent graphene quantum dots: current status and future perspectives. Nanoscale 5, 4015-4039 (2013)

3. Naik, J.P., Sutradhar, P., Saha, M.: Molecular scale rapid synthesis of graphene quantum dots (GQDs). J. Nanostruct. Chem. 7, 85-89 (2017)

4. Wu, Z., Li, W., Chen, J., Yu, C.: A graphene quantum dot-based method for the highly sensitive and selective fluorescence turn on detection of biothiols. Talanta 119, 538-543 (2014)

5. Jin, S.H., Kim, D.H., Jun, G.H., Hong, S.H., Jeon, S.: Tuning the Photoluminescence of Graphene Quantum Dots through the Charge Transfer Effect of Functional Groups. ACS Nano 7, 12391245 (2013)

6. Qu, D., Zheng, M., Zhang, L., Zhao, H., Xie, Z., Jing, X., Haddad, R.E., Fan, H., Sun, Z.: Formation mechanism and optimization of highly luminescent $\mathrm{N}$-doped graphene quantum dots. Sci. Rep. 4, 5294 (2014)

7. Pillar-Little, T., Kim, D.Y.: Differentiating the impact of nitrogen chemical states on optical properties of nitrogen-doped graphene quantum dots. RSC Adv. 7, 48263-48267 (2017)

8. Ding, W., Wei, Z., Chen, S., Qi, X., Yang, T., Hu, J., Wang, D., Wan, L.-J., Alvi, S.F., Li, L.: Space-Confinement-Induced Synthesis of Pyridinic- and Pyrrolic-Nitrogen-Doped Graphene for the Catalysis of Oxygen Reduction. Angew. Chem. Int. Ed. 52, 11755-11759 (2013)

9. Zhu, S., Song, Y., Zhao, X., Shao, J., Zhang, J., Yang, B.: The photoluminescence mechanism in carbon dots (graphene quantum dots, carbon nanodots, and polymer dots): current state and future perspective. Nano Res. 8, 355-381 (2015)

10. Achadu, O.J., Nyokong, T.: Interaction of Graphene Quantum Dots with 4-Acetamido-2,2,6,6-Tetramethylpiperidine-Oxyl Free Radicals: A Spectroscopic and Fluorimetric Study. J. Fluoresc. 26, 283-295 (2016)

11. Tetsuka, H., Asahi, R., Nagoya, A., Okamoto, K., Tajima, I., Ohta, R., Okamoto, A.: Optically Tunable Amino-Functionalized Graphene Quantum Dots. Adv. Mater. 24, 5333-5338 (2012)

12. Huang, D., Zhou, H., Wu, Y., Wang, T., Sun, L., Gao, P., Sun, Y., Huang, H., Zhou, G., Hu, J.: Bottom-up synthesis and structural design strategy for graphene quantum dots with tunable emission to the near infrared region. Carbon 142, 673-684 (2019)

13. Baker, S.N., Baker, G.A.: Luminescent Carbon Nanodots: Emergent Nanolights. Angew. Chem. Int. Ed. 49, 6726-6744 (2010)

14. Zhu, H., Wang, X., Li, Y., Wang, Z., Yang, F., Yang, X.: Microwave synthesis of fluorescent carbon nanoparticles with electrochemiluminescence properties. Chem. Commun. 34, 5118-5120 (2009)

15. Sharma, A., Das, J.: Small molecules derived carbon dots: synthesis and applications in sensing, catalysis, imaging, and biomedicine. J. Nanobiotechnology 17, 92 (2019)

16. Chen, W., Lv, G., Hu, W., Li, D., Chen, S., Dai, Z.: Synthesis and applications of graphene quantum dots: a review. Nanotechnol. Rev. 7, 157-185 (2018)

17. Dong, Y.Q., Shao, J.W., Chen, C.Q., Li, H., Wang, R.X., Chi, Y.W., Lin, X.M., Chen, G.N.: Blue luminescent graphene quantum dots and graphene oxide prepared by tuning the carbonization degree of citric acid. Carbon 50, 4738-4743 (2012)

18. Bagheri, Z., Ehtesabi, H., Rahmandoust, M., Ahadian, M.M., Hallaji, Z., Eskandari, F., Jokar, E.: New Insight into the Concept of Carbonization Degree in Synthesis of Carbon Dots to Achieve Facile Smartphone Based Sensing Platform. Sci. Rep. 7, 11013 (2017)

19. Zhao, C., Song, X., Liu, Y., Fu, Y., Ye, L., Wang, N., Wang, F., Li, L., Mohammadniaei, M., Zhang, M., Zhang, Q., Liu, J.: Synthesis of graphene quantum dots and their applications in drug delivery. J. Nanobiotechnology 18, 142 (2020)
20. Chandra, S., Das, P., Bag, S., Laha, D., Pramanik, P.: Synthesis, functionalization and bioimaging applications of highly fluorescent carbon nanoparticles. Nanoscale 3, 1533-1540 (2011)

21. de Medeiros, T.V., Manioudakis, J., Noun, F., Macairan, J.-R., Victoria, F., Naccache, R.: Microwave-assisted synthesis of carbon dots and their applications. J. Mater. Chem. C 7, 7175-7195 (2019)

22. Sharma, V., Tiwari, P., Mobin, S.M.: Sustainable carbon-dots: recent advances in green carbon dots for sensing and bioimaging. J. Mater. Chem. B 5, 8904-8924 (2017)

23. Nair, A.,Haponiuk, J. T.,Thomas, S.,Gopi, S.: Natural carbonbased quantum dots and their applications in drug delivery: A review. Biomed. Pharmacother. 132, 110834 (2020)

24. Shen, J., Shang, S., Chen, X., Wang, D., Cai, Y.: Facile synthesis of fluorescence carbon dots from sweet potato for $\mathrm{Fe} 3+$ sensing and cell imaging. Mater Sci Eng C Mater Biol Appl. 76, 856-864 (2017)

25. Wang, Y., Hu, A.: Carbon quantum dots: synthesis, properties and applications. J. Mater. Chem. C 2, 6921-6939 (2014)

26. Shin, J.H., Guo, J., Zhao, T.T., Guo, Z.X.: Functionalized Carbon Dots on Graphene as Outstanding Non-Metal Bifunctional Oxygen Electrocatalyst. Small 15, 1900296 (2019)

27. Zhang, B., Xiao, C.H., Xiang, Y., Dong, B.T., Ding, S.J., Tang, Y.H.: Nitrogen-Doped Graphene Quantum Dots Anchored on Thermally Reduced Graphene Oxide as an Electrocatalyst for the Oxygen Reduction Reaction. ChemElectroChem 3, 864-870 (2016)

28. Zhang, P., Hu, Q., Yang, X.J., Hou, X.L., Mi, J.L., Liu, L., Dong, M.D.: Size effect of oxygen reduction reaction on nitrogen-doped graphene quantum dots. RSC Adv. 8, 531-536 (2018)

29. Ye, J., Ni, K., Liu, J., Chen, G., Ikram, M., Zhu, Y.: OxygenRich Carbon Quantum Dots as Catalysts for Selective Oxidation of Amines and Alcohols. ChemCatChem 10, 259-265 (2018)

30. He, D.P., Tang, H.L., Kou, Z.K., Pan, M., Sun, X.L., Zhang, J.J., Mu, S.C.: Engineered Graphene Materials: Synthesis and Applications for Polymer Electrolyte Membrane Fuel Cells. Adv. Mater. 29, 1601741 (2017)

31. Lim, S.Y., Shen, W., Gao, Z.: Carbon quantum dots and their applications. Chem. Soc. Rev. 44, 362-381 (2015)

32. Wu, Z.L., Zhang, P., Gao, M.X., Liu, C.F., Wang, W., Leng, F., Huang, C.Z.: One-pot hydrothermal synthesis of highly luminescent nitrogen-doped amphoteric carbon dots for bioimaging from Bombyx mori silk - natural proteins. J. Mater. Chem. B 1, 2868-2873 (2013)

33. Barbooti, M.M., Al-Sammerrai, D.A.: Thermal decomposition of citric acid. Thermochim. Acta 98, 119-126 (1986)

34. Wang, S., Chen, Z.-G., Cole, I., Li, Q.: Structural evolution of graphene quantum dots during thermal decomposition of citric acid and the corresponding photoluminescence. Carbon $\mathbf{8 2}$, 304-313 (2015)

35. Hill, S., Galan, M.C.: Fluorescent carbon dots from mono- and polysaccharides: synthesis, properties and applications. Beilstein J. Org. Chem. 13, 675-693 (2017)

36. Klinger, K.M., Liebner, F., Fritz, I., Potthast, A., Rosenau, T.: Formation and Ecotoxicity of N-Heterocyclic Compounds on Ammoxidation of Mono- and Polysaccharides. J. Agric. Food Chem. 61, 9004-9014 (2013)

37. Demir-Cakan, R., Baccile, N., Antonietti, M., Titirici, M.-M.: Carboxylate-Rich Carbonaceous Materials via One-Step Hydrothermal Carbonization of Glucose in the Presence of Acrylic Acid. Chem. Mater. 21, 484-490 (2009)

38. Peng, H., Li, Y., Jiang, C., Luo, C., Qi, R., Huang, R., Duan, C.-G., Travas-Sejdic, J.: Tuning the properties of luminescent nitrogen-doped carbon dots by reaction precursors. Carbon 100, 386-394 (2016) 
39. Tang, L.B., Ji, R.B., Cao, X.K., Lin, J.Y., Jiang, H.X., Li, X.M., Teng, K.S., Luk, C.M., Zeng, S.J., Hao, J.H., Lau, S.P.: Deep Ultraviolet Photoluminescence of Water-Soluble Self-Passivated Graphene Quantum Dots. ACS Nano 6, 5102-5110 (2012)

40. Yang, Z.-C., Li, X., Wang, J.: Intrinsically fluorescent nitrogencontaining carbon nanoparticles synthesized by a hydrothermal process. Carbon 49, 5207-5212 (2011)

41. Barzetti, T.,Selli, E.,Moscotti, D.,Forni, L.: Pyridine and ammonia as probes for FTIR analysis of solid acid catalysts. J. Chem. Soc., Faraday Trans. 92, 1401-1407 (1996)

42. Randhawa, H.S., Kapoor, J.L.: CN Stretching frequencies in amide systems. J. Comput. Chem. 2, 12-13 (1981)

43. Lazar, P., Mach, R., Otyepka, M.: Spectroscopic Fingerprints of Graphitic, Pyrrolic, Pyridinic, and Chemisorbed Nitrogen in N-Doped Graphene. J. Phys. Chem. C 123, 10695-10702 (2019)

44. Tian, L., Ghosh, D., Chen, W., Pradhan, S., Chang, X.J., Chen, S.W.: Nanosized Carbon Particles From Natural Gas Soot. Chem. Mater. 21, 2803-2809 (2009)

45. Liu, H.P., Ye, T., Mao, C.D.: Fluorescent carbon nanoparticles derived from candle soot. Angew. Chem. Int. Ed. 46, 6473-6475 (2007)

46. Song, L.Q., Shi, J.J., Lu, J., Lu, C.: Structure observation of graphene quantum dots by single-layered formation in layered confinement space. Chem. Sci. 6, 4846-4850 (2015)

47. Linehan, K., Doyle, H.: Size controlled synthesis of carbon quantum dots using hydride reducing agents. J. Mater. Chem. C 2, 6025-6031 (2014)

48. Rigodanza, F., Burian, M., Arcudi, F., Đorđević, L., Amenitsch, H., Prato, M.: Snapshots into carbon dots formation through a combined spectroscopic approach. Nat. Commun. 12, 2640 (2021)

49. Ewels, C.P., Glerup, M.: Nitrogen doping in carbon nanotubes. J. Nanosci. Nanotechnol. 5, 1345-1363 (2005)

50. Simoes, E. F. C.,Esteves da Silva, J. C. G.,Leitao, J. M. M.: Carbon dots from tryptophan doped glucose for peroxynitrite sensing. Anal. Chim. Acta 852, 174-180 (2014)

51. Mazzotta, E., Rella, S., Turco, A., Malitesta, C.: XPS in development of chemical sensors. RSC Adv. 5, 83164-83186 (2015)

52. Chen, Y., Xie, B., Ren, Y., Yu, M., Qu, Y., Xie, T., Zhang, Y., Wu, Y.: Designed nitrogen doping of few-layer graphene functionalized by selective oxygenic groups. Nanoscale Res. Lett. 9, 646 (2014)

53. Wang, D., Wang, Z.Y., Zhan, Q.Q., Pu, Y., Wang, J.X., Foster, N.R., Dai, L.M.: Facile and Scalable Preparation of Fluorescent Carbon Dots for Multifunctional Applications. Eng. J. 3, 402-408 (2017)

54. Senel, B., Demir, N., Buyukkoroglu, G., Yildiz, M.: Graphene quantum dots: Synthesis, characterization, cell viability, genotoxicity for biomedical applications. Saudi Pharm. J. 27, 846-858 (2019)

55. Becerra-Arciniegas, R. A.,Narducci, R.,Ercolani, G.,Antonaroli, S.,Sgreccia, E.,Pasquini, L.,Knauth, P.,Di Vona, M. L.: Alkaline stability of model anion exchange membranes based on poly (phenylene oxide) (PPO) with grafted quaternary ammonium groups: Influence of the functionalization route. Polymer 185, 121931 (2019)

56. Ding, H.,Li, X. H.,Chen, X. B.,Wei, J. S.,Li, X. B.,Xiong, H. M.: Surface states of carbon dots and their influences on luminescence. J. Appl. Phys. 127, 231101 (2020)
57. Ciotta, E., Prosposito, P., Tagliatesta, P., Lorecchio, C., Stella, L., Kaciulis, S., Soltani, P., Placidi, E., Pizzoferrato, R.: Discriminating between Different Heavy Metal Ions with Fullerene-Derived Nanoparticles. Sensors 18, 1496 (2018)

58. Lin, L.X., Zhang, S.W.: Creating high yield water soluble luminescent graphene quantum dots via exfoliating and disintegrating carbon nanotubes and graphite flakes. Chem. Commun. 48, 10177-10179 (2012)

59. Ciotta, E., Prosposito, P., Pizzoferrato, R.: Positive curvature in Stern-Volmer plot described by a generalized model for static quenching. J. Lumin. 206, 518-522 (2019)

60. Zhang, C.F., Cui, Y.Y., Song, L., Liu, X.F., Hu, Z.B.: Microwave assisted one-pot synthesis of graphene quantum dots as highly sensitive fluorescent probes for detection of iron ions and $\mathrm{pH}$ value. Talanta 150, 54-60 (2016)

61. Sun, J., Yang, S.W., Wang, Z.Y., Shen, H., Xu, T., Sun, L.T., Li, H., Chen, W.W., Jiang, X.Y., Ding, G.Q., Kang, Z.H., Xie, X.M., Jiang, M.H.: Ultra-High Quantum Yield of Graphene Quantum Dots: Aromatic-Nitrogen Doping and Photoluminescence Mechanism. Part. Part. Syst. Charact. 32, 434-440 (2015)

62. Zhang, X.X., Zhang, W.J., Tang, S.K.: L-Tryptophan functionalized graphene quantum dots as a fluorescence indicator for $\mathrm{pH}$ detection in real water. J. Photochem. Photobiol. A 372, 71-77 (2019)

63. Zhao, H.G., Liu, G.J., You, S.J., Camargo, F.V.A., Zavelani-Rossi, M., Wang, X.H., Sun, C.C., Liu, B., Zhang, Y.M., Han, G.T., Vomiero, A., Gong, X.: Gram-scale synthesis of carbon quantum dots with a large Stokes shift for the fabrication of eco-friendly and high-efficiency luminescent solar concentrators. Energy Environ. Sci. 14, 12 (2021)

64. Kausar, A.: Polymer/carbon-based quantum dot nanocomposite: forthcoming materials for technical application. J. Macromol. Sci. A 56, 341-356 (2019)

65. Ding, Y., Zhang, F., Xu, J., Miao, Y., Yang, Y., Liu, X., Xu, B.: Synthesis of short-chain passivated carbon quantum dots as the light emitting layer towards electroluminescence. RSC Adv. 7, 28754-28762 (2017)

66. Deng, X., Feng, Y., Li, H., Du, Z., Teng, Q., Wang, H.: N-doped carbon quantum dots as fluorescent probes for highly selective and sensitive detection of Fe3+ ions. Particuology 41, 94-100 (2018)

67. Hou, H.S., Banks, C.E., Jing, M.J., Zhang, Y., Ji, X.B.: Carbon Quantum Dots and Their Derivative 3D Porous Carbon Frameworks for Sodium-Ion Batteries with Ultralong Cycle Life. Adv. Mater. 27, 7861-7866 (2015)

68. Wu, J., Ma, S., Sun, J., Gold, J.I., Tiwary, C., Kim, B., Zhu, L., Chopra, N., Odeh, I.N., Vajtai, R., Yu, A.Z., Luo, R., Lou, J., Ding, G., Kenis, P.J.A., Ajayan, P.M.: A metal-free electrocatalyst for carbon dioxide reduction to multi-carbon hydrocarbons and oxygenates. Nat. Commun. 7, 13869 (2016)

Publisher's Note Springer Nature remains neutral with regard to jurisdictional claims in published maps and institutional affiliations. 